Eilbeck, J. C., V. Z. Enolski, S. Matsutani, Y. Ônishi, and E. Previato. (2007) “Abelian Functions for Trigonal Curves of Genus Three,"

International Mathematics Research Notices, Vol. 2007, Article ID rnm140, 38 pages.

doi:10.1093/imrn/rnm140

\title{
Abelian Functions for Trigonal Curves of Genus Three
}

\section{J. C. Eilbeck, ${ }^{1}$ V. Z. Enolski, ${ }^{2}$ S. Matsutani, ${ }^{3}$ Y. Ônishi ${ }^{4}$ and E. Previato ${ }^{5}$}

${ }^{1}$ Department of Mathematics and the Maxwell Institute for Mathematical Sciences, Heriot-Watt University, Edinburgh, EH14 4AS UK, ${ }^{2}$ Institute of Magnetism, Vernadski blvd. 36, Kiev-142, Ukraine, ${ }^{3} 8-21-1$ Higashi-Linkan, Sagamihara, 228-0811, Japan, ${ }^{4}$ Faculty of Humanities and Social Sciences, Iwate University, Ueda 3-18-34, Morioka 020-8550, Japan, and ${ }^{5}$ Department of Mathematics and Statistics, Boston University, Boston, MA 02215-2411, USA

Correspondence to be sent to: J. C. Eilbeck, Department of Mathematics and the Maxwell Institute for Mathematical Sciences, Heriot-Watt University, Edinburgh, UK EH14 4AS.

e-mail: J.C.Eilbeck@hw.ac.uk

We develop the theory of generalized Weierstrass $\sigma$-and $\wp$-functions defined on a general trigonal curve of genus three. In particular, we give a list of the associated partial differential equations satisfied by the $\wp$-functions, a proof that the coefficients of the power series expansion of the $\sigma$-function are polynomials of coefficients of the defining equation of the curve, and the derivation of two addition formulae.

\section{Introduction}

Constructive theories of Abelian and modular functions associated with algebraic curves have seen an upsurge of interest in recent times. These classical functions have been of crucial importance in mathematics since their definition at the hands of Abel,

Received September 28, 2006; Revised October 9, 2007; Accepted October 19, 2007

See http://www.oxfordjournals.org/our_journals/imrn/for proper citation instructions.

(C) The Author 2007. Published by Oxford University Press. All rights reserved. For permissions, please e-mail:journals.permissions@oxfordjournals.org. 
Jacobi, Poincaré and Riemann, but their relevance in physics and applied mathematics has greatly developed over the past three decades. Algebraic curves are here intended as Riemann surfaces, unless specified to be singular.

The study of the simplest hyperelliptic curves, namely curves of genus two, goes back to the beginning of the 20th century, and these are treated in much detail in advanced textbooks, see for example Baker (1907) [4] and more recently Cassels and Flynn (1996) [12]. Not so much is known about the simplest trigonal curves, which have genus three. The study of modular functions of these curves was originated by Picard, and reprised recently by Shiga [27] and his school. In this article we study Abelian functions associated with the general non-singular $(3,4)$-curve. This is an $(n, m)$-curve in the sense of Burchnall-Chaundy [11]

Our work is based on the realization of Abelian functions as logarithmic derivatives of the multi-dimensional $\sigma$-function. This approach is due to Weierstrass and Klein and was developed by Baker [1]; for recent developments of the theory of multidimensional $\sigma$-functions, see Grant [19], Buchstaber, Enolskii, and Leykin [9], Buchstaber and Leykin [7, 8], Eilbeck, Enolskii and Previato [17], Baldwin and Gibbons [5], and Ônishi and Matsutani [26, 25] among others.

We shall adopt as a template the Weierstrass theory of elliptic functions, trying to extend as far as possible these results to the case of the trigonal genus-three curve. Let $\sigma(u)$ and $\wp(u)$ be the standard functions in Weierstrass elliptic function theory. They satisfy the well-known formulae

$$
\wp(u)=-\frac{d^{2}}{d u^{2}} \log \sigma(u), \quad\left(\wp^{\prime}\right)^{2}=4 \wp^{3}-g_{2} \wp-g_{3}, \quad \wp^{\prime \prime}=6 \wp^{2}-\frac{1}{2} g_{2}
$$

and the addition formula, which is a basic formula of the theory

$$
-\frac{\sigma(u+v) \sigma(u-v)}{\sigma(u)^{2} \sigma(v)^{2}}=\wp(u)-\wp(v) .
$$

We present here two additional formulae (Theorems 9.1 and 10.1). The first of these is for the general trigonal curve of degree four, whereas the second is restricted to a "purely trigonal" curve of degree four (see Equation (3.1)). The first main Theorem 9.1 is the natural generalization of Equation (1.2). The authors realized the existence of the formula of the second main Theorem 10.1 from [25]. However we were not able to use that article to establish our result, instead working from results by Cho and Nakayashiki [13], Grant's paper [19], p. 100, (1.6), or a calculation using [10]. The crucial part is to identify the coefficients of the right hand sides of these two formulae. To calculate these, we used 
a power-series expansion of the $\sigma$-function, stimulated by the works of Buchstaber and Leykin [7] for hyperelliptic case and of Baldwin and Gibbons [5] for a purely trigonal curve of genus four.

The $\sigma$-functional realization of Abelian functions of trigonal curve of arbitrary genus $g$ was previously developed in [10] and [16]. Using these results in the case of $g=3$ we present explicit formulae for six canonical meromorphic differentials and the symmetric bi-differential which allow us to derive a set of relations for trigonal $\wp$ functions, generalizing the above relations for the Weierstrass $\wp$-function.

Note that we have recently developed a parallel, but more limited theory, for purely trigonal curves of genus four in [6], a paper which draws heavily on the results presented here. It is perhaps useful to compare and contrast these two cases. As demonstrated in Schilling's generalization of the Neumann system [28], there are basically two cases of trigonal cyclic covers, the order of a related linear differential operator that commutes with the given one of order three being congruent to 1 or 2 modulo 3 , respectively. In each case, the action variables of the integrable system parameterize a family of curves of the same type, thus the family of curves in the $(3,4)$-case cannot be obtained as a limit of that in the $(3,5)$-case, as they have different dimensions. In the present article, we develop the method and prove the addition formulae, together with the characterizing differential equations, for the former case, in that the highest power of $x$ appearing in the equation of the curve is 4 (三1 modulo 3 ); this corresponds to the "base" case of the Boussinesq equation, the smallest-genus spectral curve of an algebro-geometric third-order operator. In [6], the case where the highest power of $x$ appearing in the equation of the curve is 5 ( $\equiv 2$ modulo 3 ) is addressed. The differences in the two cases manifest themselves in a number of ways, for example the parity of the $\sigma$-function is different in the two cases, and the two-term addition formulae are antisymmetric in the genus 3 case and symmetric in the genus 4 case. Also the results are given for the general $(3,4)$-curve here, whereas only for the purely trigonal $(3,5)$-case in [6]. It may be possible with some work to relate the $(3,5)$ case to the $(3,4)$-case, but this would not be straightforward and we have not yet attempted this.

Our study is far from complete, and a number of questions still remain. One of the first problems still to be considered should be the explicit recursive construction of the $\sigma$-series generalizing the one given by Weierstrass; for a hyperelliptic curve of genus two, this result was found by Buchstaber and Leykin [7], who also devised a 
procedure to derive such recursions for the whole family of $(n, m)$-curves [7], [8]. Another problem is the deeper understanding of the algebraic structure of the addition theorems developed here, in order to generalize results to higher genera. As a pattern one can consider the addition formula of [9] for hyperelliptic $\sigma$-functions of arbitrary genera written in terms of certain Pfaffians. Also, the description of Jacobi and Kummer varieties as projective varieties, whose coordinates are given in terms of (derivatives of) trigonal $\wp$-functions, is far from complete. We hope the results we present to be the first steps towards a general theory of trigonal curves of arbitrary genus, as well as a tool in the study of projective varieties which are images of Jacobians.

The article is organized as follows. We first discuss the basic properties of the general $(3,4)$-curve in Section 2, and define a restricted version of this curve, the "purely trigonal case", in Section 3. In Section 4, we introduce the $\sigma$-function for the general curve, and in Section 5 the Abelian functions $\wp_{i j}$ and their derivatives. Section 6 of the article is devoted to the various differential relations satisfied by these Abelian functions, and the series expansion of the $\sigma$-function is discussed in Section 7, in which the result (Theorem 7.1) is new, is proved quite constructively, and is the key for the rest of the article. Let $\Theta^{[2]}$ be the standard theta divisor, namely the image of the Abelian map of the symmetric square of the curve that we consider, in its Jacobian variety $J$. The basis of the spaces $\Gamma\left(J, \mathcal{O}\left(n \Theta^{[2]}\right)\right)$ of functions on $J$ whose poles are at most of order $n$ along $\Theta^{[2]}$ are discussed in Section 8, as a preliminary to the two main addition Theorems in Sections 9 and 10, respectively. The first addition theorem is a two-term relation for the general $(3,4)$-curve, and the second a three-term relation for the "purely trigonal" $(3,4)$ curve. Appendix A has some formulae for the fundamental bi-differential, and Appendix B has a list of quadratic 3-index relations for the "purely trigonal" case only, as the full relations would require too much space. The web site [15] contains more details of the relations omitted through lack of space.

While Sections 2 and 3 overlap somewhat with material in [25], we believe that the results are useful to make the present article reasonably self-contained.

\section{Trigonal Curves of Genus Three}

Let $C$ be the curve defined by $f(x, y)=0$, where

$$
\begin{aligned}
f(x, y)=y^{3}+ & \left(\mu_{1} x+\mu_{4}\right) y^{2}+\left(\mu_{2} x^{2}+\mu_{5} x+\mu_{8}\right) y \\
& -\left(x^{4}+\mu_{3} x^{3}+\mu_{6} x^{2}+\mu_{9} x+\mu_{12}\right), \quad\left(\mu_{j} \text { are constants }\right)
\end{aligned}
$$


with the unique point $\infty$ at infinity. This curve is of genus 3 , if it is nonsingular. We consider the set of 1 -forms

$$
\omega_{1}=\frac{d x}{f_{Y}(x, y)}, \quad \omega_{2}=\frac{x d x}{f_{Y}(x, y)}, \quad \omega_{3}=\frac{y d x}{f_{Y}(x, y)},
$$

where $f_{Y}(x, y)=\frac{\partial}{\partial y} f(x, y)$. This is a basis of the space of differentials of the first kind on $C$. We denote the vector consisting of the forms (2.2) by

$$
\omega=\left(\omega_{1}, \omega_{2}, \omega_{3}\right)
$$

We know, by the general theory, that for three variable points $\left(x_{1}, y_{1}\right),\left(x_{2}, y_{2}\right)$, and $\left(x_{3}, y_{3}\right)$ on $C$, the sum of integrals from $\infty$ to these three points

$$
\begin{aligned}
u & =\left(u_{1}, u_{2}, u_{3}\right) \\
& =\int_{\infty}^{\left(x_{1}, Y_{1}\right)} \omega+\int_{\infty}^{\left(x_{2}, Y_{2}\right)} \omega+\int_{\infty}^{\left(x_{3}, Y_{3}\right)} \omega
\end{aligned}
$$

fills the whole space $\mathbb{C}^{3}$. We denote the points in $\mathbb{C}^{3}$ by $u$ and $v$ etc., and their natural coordinates in $\mathbb{C}^{3}$ by the subscripts $\left(u_{1}, u_{2}, u_{3}\right),\left(v_{1}, v_{2}, v_{3}\right)$. We denote the lattice generated by the integrals of the vector (2.3) along any closed paths on $C$ by $\Lambda$. We denote the manifold $\mathbb{C}^{3} / \Lambda$, by $J$, the Jacobian variety over $\mathbb{C}$ of $C$. We denote by $\kappa$ the natural map to the quotient group,

$$
\kappa: \mathbb{C}^{3} \rightarrow \mathbb{C}^{3} / \Lambda=J
$$

We have $\Lambda=\kappa^{-1}((0,0,0))$. We define for $k=1,2,3, \ldots$, the map

$$
\begin{aligned}
& \iota: \operatorname{Sym}^{k}(C) \rightarrow J \\
& \left(P_{1}, \cdots, P_{k}\right) \mapsto\left(\int_{\infty}^{P_{1}} \omega+\cdots+\int_{\infty}^{P_{k}} \omega\right) \bmod \Lambda,
\end{aligned}
$$

and denote its image by $W^{[k]}$. ( $W^{[k]}=J$ for $k \geq 3$ by the Abel-Jacobi theorem.) Let

$$
[-1]\left(u_{1}, u_{2}, u_{3}\right)=\left(-u_{1},-u_{2},-u_{3}\right)
$$

and

$$
\Theta^{[k]}:=W^{[k]} \cup[-1] W^{[k]}
$$


We call this $\Theta^{[k]}$ the $k$-th standard theta subset. In particular, if $k=1$, then (2.6) gives an embedding of $C$ :

$$
\begin{aligned}
\iota: C \rightarrow J \\
P \mapsto \int_{\infty}^{P} \omega \bmod \wedge .
\end{aligned}
$$

We note that

$$
\Theta^{[2]}=W^{[2]}, \quad \Theta^{[1]} \neq W^{[1]}
$$

differing from the genus-3 hyperelliptic case in a suitable normalization [9]. If $u=$ $\left(u_{1}, u_{2}, u_{3}\right)$ varies on the inverse image $\kappa^{-1} \iota(C)=\kappa^{-1}\left(W^{[1]}\right)$ of the embedded curve, we can take $u_{3}$ as a local parameter at the origin $(0,0,0)$. Then we have (see [25], e.g.) Laurent expansions with respect to $u_{3}$ as follows:

$$
u_{1}=\frac{1}{5} u_{3}^{5}+\cdots, \quad u_{2}=\frac{1}{2} u_{3}^{2}+\cdots
$$

and

$$
x(u)=\frac{1}{u_{3}^{3}}+\cdots, \quad y(u)=\frac{1}{u_{3}^{4}}+\cdots .
$$

We introduce a weight for several variables as follows:

DEFINITION 2.1. We define a weight for constants and variables appearing in our relations as follows. The weights of the variables $u_{1}, u_{2}, u_{3}$ for every $u=\left(u_{1}, u_{2}, u_{3}\right)$ of $W^{[k]},(k=1,2, \ldots)$ are $5,2,1$, respectively, and the weight of each coefficient $\mu_{j}$ in $(2.1)$ is $-j$, the weights of $x$ and $y$ of each point $(x, y)$ of $C$ are -3 and -4 , respectively. So, the weights of the variables are nothing but the order of zero at $\infty$, while the weight assigned to the coefficients is a device to render $f(x, y)$ homogeneous. This is the reason why $\mu_{7}$, $\mu_{10}, \mu_{11}$ are absent.

We remark that the weights of the variables $u_{k}$ are precisely the Weierstrass gap numbers of the Weierstrass gap sequence at $\infty$, whilst the weights of monomials of $x(u)$ and $y(u)$ correspond to the Weierstrass nongap numbers in the sequence. In particular, in the case considered the Weierstrass gap sequence is of the form

$$
\overline{0}, 1,2, \overline{3,4}, 5, \overline{6,7,8,9,10, \ldots}
$$

where orders of existing functions of the form $x^{p} y^{q}, p, q \in \mathbb{N} \cup\{0\}$ are overlined. 
The definition above is compatible, for instance, with the Laurent expansion of $x(u)$ and $y(u)$ with respect to $u_{3}$, etc. for $u \in W^{[1]}$. Moreover, all the equalities in this article are homogeneous with respect to this weight.

In the next section, we use the discriminant of $C$. Heuristically, the discriminant $D$ of $C$ is defined as (one of) the simplest polynomial(s) in the $\mu_{j}$ 's such that $D=0$ if and only if $C$ has a singular point. Here we are regarding $C$ as a family of curves over $\mathbb{Z}$. While no concrete expression of the discriminant is necessary for the main results in this article, we put forward a conjecture based on the results of experimentation on special cases of $C$ using computer algebra.

CONJECTURE 2.2. Let

$$
\begin{aligned}
& R_{1}=\operatorname{rslt}_{x}\left(\operatorname{rslt}_{Y}\left(f(x, y), f_{x}(x, y)\right), \operatorname{rslt}_{Y}\left(f(x, y), f_{Y}(x, y)\right)\right), \\
& R_{2}=\operatorname{rslt}_{Y}\left(\operatorname{rslt}_{x}\left(f(x, y), f_{x}(x, y)\right), \operatorname{rslt}_{x}\left(f(x, y), f_{Y}(x, y)\right)\right), \\
& R_{3}=\operatorname{gcd}\left(R_{1}, R_{2}\right),
\end{aligned}
$$

where rslt $_{z}$ represents the resultant, namely, the determinant of the Sylvester matrix with respect to the variable $z$. Then $R_{3}$ is of weight 144 and a perfect square in the ring

$$
\mathbb{Z}\left[\mu_{1}, \mu_{4}, \mu_{2}, \mu_{5}, \mu_{8}, \mu_{3}, \mu_{6}, \mu_{9}, \mu_{12}\right] .
$$

Unfortunately checking this condition directly is a computing task presenting considerable difficulties due to the size of the intermediate expressions involved. We leave this as a conjecture and remark only that work on a full calculation is continuing. This result is not crucial to this paper, but we will adopt it as a working hypothesis (see Remark 7.2). To continue, we define here the discriminant $D$ of $C$ by a square root of $R_{3}$ :

$$
D=\sqrt{R_{3}} .
$$

We comment on the choice of this root in Remark 7.2. If the conjecture is true, $D$ is of weight 72. For the convenience of the reader we give $R_{3}$

$$
\begin{aligned}
R_{3}= & \left(256 \mu_{12}{ }^{3}-27 \mu_{12}{ }^{2} \mu_{3}{ }^{4}-128 \mu_{12}{ }^{2} \mu_{6}{ }^{2}+144 \mu_{12}{ }^{2} \mu_{6} \mu_{3}{ }^{2}-192 \mu_{12}{ }^{2} \mu_{9} \mu_{3}+16 \mu_{12} \mu_{6}{ }^{4}\right. \\
& -80 \mu_{12} \mu_{9} \mu_{6}{ }^{2} \mu_{3}-4 \mu_{12} \mu_{3}{ }^{2} \mu_{6}{ }^{3}+18 \mu_{12} \mu_{9} \mu_{3}{ }^{3} \mu_{6}+144 \mu_{12} \mu_{9}{ }^{2} \mu_{6}-6 \mu_{12} \mu_{9}{ }^{2} \mu_{3}{ }^{2} \\
& \left.-4 \mu_{9}{ }^{2} \mu_{6}{ }^{3}-4 \mu_{9}{ }^{3} \mu_{3}{ }^{3}+\mu_{9}{ }^{2} \mu_{3}{ }^{2} \mu_{6}{ }^{2}+18 \mu_{9}{ }^{3} \mu_{6} \mu_{3}-27 \mu_{9}^{4}\right)^{6}
\end{aligned}
$$

for the special case of $\mu_{1}=\mu_{2}=\mu_{4}=\mu_{5}=\mu_{8}=0$ (see Section 3). 
DEFINITION 2.3. The 2-form $\Omega((x, y),(z, w))$ on $C \times C$ is called fundamental 2 -from of the second kind or (fundamental second kind bi-differential) if it is symmetric, namely,

$$
\Omega((x, y),(z, w))=\Omega((z, w),(x, y)),
$$

it has its only pole (of second order) along the diagonal of $C \times C$, and in the vicinity of each point $(x, y)$ it is expanded in power series as

$$
\Omega((x, y),(z, w))=\left(\frac{1}{\left(\xi-\xi^{\prime}\right)^{2}}+O(1)\right) d \xi d \xi^{\prime} \quad(\text { as }(x, y) \rightarrow(z, w)),
$$

where $\xi$ and $\xi^{\prime}$ are local coordinates of points $(x, y)$ and $(z, w)$.

We shall look for a realization of $\Omega((x, y),(z, w))$ in the form

$$
\Omega((x, y),(z, w))=\frac{F((x, y),(z, w)) d x d z}{(x-z)^{2} f_{y}(x, y) f_{w}(z, w)}
$$

where $F((x, y),(z, w))$ is a polynomial in its variables.

LEMMA 2.4 (Fundamental 2-form of the second kind). Let $\Sigma((x, y),(z, w))$ be the meromorphic function on $C \times C$,

$$
\Sigma((x, y),(z, W))=\left.\frac{1}{(x-z) f_{Y}(x, y)} \sum_{k=1}^{3} y^{3-k}\left[\frac{f(Z, W)}{W^{3-k+1}}\right]_{W}\right|_{(Z, W)=(z, W)},
$$

where [ ] $]_{W}$ means removing the terms of negative powers with respect to $W$. Then there exist differentials $\eta_{j}=\eta_{j}(x, y)(j=1,2,3)$ of the second kind that have their only pole at $\infty$ such that the fundamental 2-form of the second kind is given as ${ }^{1}$,

$$
\Omega((x, y),(z, w))=\left(\frac{d}{d x} \Sigma((z, w),(x, y))+\sum_{k=1}^{3} \frac{\omega_{k}(z, w)}{d z} \frac{\eta_{k}(x, y)}{d x}\right) d x d z
$$

The set of differentials $\left\{\eta_{1}, \eta_{2}, \eta_{3}\right\}$ is determined modulo the space spanned by the $\omega_{j}$ s of (2.2).

PROOF. The 2-form

$$
\frac{d}{d z} \Sigma((x, y),(z, w)) d x d z
$$

\footnotetext{
${ }^{1}$ Since $x$ and $y$ are related, we do not use $\partial$.
} 
satisfies the condition on the poles as a function of $(x, y)$, indeed one can check that (2.20) has only a second order pole at $(x, y)=(z, W)$ whenever $(z, W)$ is an ordinary point or a Weierstrass point; at infinity the expansion (2.12) should be used. However, the form (2.20) has unwanted poles at infinity as a form in the $(z, w)$-variables. To restore the symmetry given in (2.15) we complement (2.20) by the second term to obtain (2.19) with polynomials $\eta_{j}(x, y)$ which should be found from (2.15). That results in a system of linear equations for coefficients of $\eta_{j}(x, y)$ which is always solvable. As a result, the polynomials $\eta_{i}(x, y)$ as well as $F((x, y),(z, w))$ are obtained explicitly.

REMARK 2.5. The 1 -form

$$
\Pi_{\left(z_{1}, W_{1}\right)}^{\left(z_{2}\right)}(x, y)=\Sigma\left((x, y),\left(z_{1}, w_{1}\right)\right) d x-\Sigma\left((x, y),\left(z_{2}, w_{2}\right)\right) d x
$$

is the differential of the third kind, with first order poles at points $(x, y)=\left(z_{1}, w_{1}\right)$ and $(x, y)=\left(z_{2}, w_{2}\right)$, and residues +1 and -1 correspondingly.

REMARK 2.6. The realization of the fundamental 2-form in terms of the Schottky-Klein prime-form and $\theta$-functions is given in [1], no. 272, and the theory based on the $\theta$-functional representation is developed in [18]. Here we deal with an equivalent algebraic representation of the fundamental 2-form which goes back to Klein and exhibit an algebraic expression for it, that is also mentioned by Fay in [18] where the primeform was defined. The above derivation of the fundamental 2-form is done in [1], around page 194, and it was reconsidered in [16] for a large family of algebraic curves. The case of a trigonal curve of genus four was developed in [5], pp. 3617-3618.

It is easily seen that the $\eta_{j}$ above is written as

$$
\eta_{j}(x, y)=\frac{h_{j}(x, y)}{f_{Y}(x, y)} d x, \quad j=1,2,3
$$

where $h_{j}(x, y) \in \mathbb{Q}\left[\mu_{1}, \mu_{2}, \mu_{4}, \mu_{5}, \mu_{8}, \mu_{3}, \mu_{6}, \mu_{9}, \mu_{12}\right][x, y]$, and $h_{j}$ is of homogeneous weight.

The differentials $\eta_{j}$ are defined modulo the space of holomorphic differentials with the same weight, but it is possible to choose the standard $\eta_{j}$ s uniquely by requiring that for each $j=1,2,3$ the polynomial $h_{j}(x, y)$ do not contain monomials corresponding to nongaps with bigger $j$. Moreover there exist precisely $2 g=6$ monomials defining standard differentials, for more details see [8], Chapter 4. 
In particular, straightforward calculations lead to the following expressions

$$
\begin{aligned}
h_{3}(x, y)= & -x^{2}, \\
h_{2}(x, y)= & -2 x y+\mu_{1} x^{2}, \\
h_{1}(x, y)= & -\left(5 x^{2}+\left(\mu_{1} \mu_{2}-3 \mu_{3}\right) x+\mu_{2} \mu_{4}+\mu_{6}\right) y+\mu_{2} y^{2}+3 \mu_{1} x^{3} \\
& -\left(\mu_{2}{ }^{2}+2 \mu_{3} \mu_{1}-2 \mu_{4}\right) x^{2}-\left(\mu_{5} \mu_{2}+\mu_{6} \mu_{1}+\mu_{3} \mu_{4}\right) x+\frac{3}{4} \mu_{1} f_{x}(x, y) \\
& -\left(\frac{1}{3} \mu_{2}-\frac{1}{4} \mu_{1}^{2}\right) f_{Y} .
\end{aligned}
$$

The orders of monomials defining standard differentials are printed in bold:

$$
\overline{\mathbf{0}}, \mathbf{1}, 2, \overline{\mathbf{3}, \mathbf{4}}, 5, \overline{\mathbf{6}, \mathbf{7}, 8,9, \mathbf{1 0}, \ldots}
$$

these can be written as $3 i+4 j, 0 \leq i \leq 2,0 \leq j \leq 1$. We remark that the last two terms in the definition of $h_{1}(x, y)$ are chosen to provide the standard differentials described above. The polynomial $F((x, y),(z, w))$ in (2.17) is of homogeneous weight (weight -8$)$, and is given explicitly in Appendix A.

\section{Purely Trigonal Curve of Degree Four}

In Section 10 of this article, we restrict ourselves to the curve

$$
C: y^{3}=x^{4}+\mu_{3} x^{3}+\mu_{6} x^{2}+\mu_{9} x+\mu_{12}
$$

specialized from (2.1). We also restrict results given in Appendix B to this case to save space. This curve is called a purely trigonal curve of degree four. Equivalently we can represent the curve (2.1) in the form

$$
C: y^{3}=\prod_{k=1}^{4}\left(x-a_{k}\right)
$$

and evaluate the discriminant $D$ according to (2.14) as

$$
D=\prod_{1 \leq i<j \leq 4}\left(a_{i}-a_{j}\right)^{4}
$$

The curve $C$ is smooth if and only if $a_{i} \neq a_{j}$ for all $i, j=1, \ldots 4$. While we assume this to be the case, results in the singular cases are obtained by suitable limiting process. 
For the curve (3.1), the basis (2.2) of differential forms of first kind and the function $\Sigma$ in (2.18) can be written explicitly as

$$
\omega_{1}=\frac{d x}{3 y^{2}}, \quad \omega_{2}=\frac{x d x}{3 y^{2}}, \quad \omega_{3}=\frac{y d x}{3 y^{2}}=\frac{d x}{3 y}
$$

and

$$
\Sigma((x, y),(z, w))=\frac{y^{2}+y w+w^{2}}{3(x-z) y^{2}}
$$

respectively. The function $\sigma(u)$ is defined by using these. Let

$$
\zeta=e^{2 \pi \sqrt{-1} / 3}
$$

The curve $C$ has an automorphism $(x, y) \mapsto(x, \zeta y)$, and for $u=\left(u_{1}, u_{2}, u_{3}\right) \in \kappa^{-1} \iota(C), \zeta^{j}$ acts by

$$
\left[\zeta^{j}\right] u=\left(\zeta^{j} u_{1}, \zeta^{j} u_{2}, \zeta^{2 j} u_{3}\right)=\int_{\infty}^{\left(x, \zeta^{j} y\right)}\left(d u_{1}, d u_{2}, d u_{3}\right)
$$

This action naturally induces an action on $\kappa^{-1} \Theta^{[k]},(k=2,3, \ldots)$, implying that the set $\Theta^{[k]}$ is stable under the action of $\left[\zeta^{j}\right]$.

\section{The $\sigma$-function}

We construct here the $\sigma$-function

$$
\sigma(u)=\sigma\left(u_{1}, u_{2}, u_{3}\right)
$$

associated with $C$ for $u \in \mathbb{C}^{3}$ (see also [9], Chapter 1 ). We choose closed paths

$$
\alpha_{i}, \beta_{j}(1 \leqq i, j \leqq 3)
$$

on $C$ which generate $H_{1}(C, \mathbb{Z})$ such that their intersection numbers are $\alpha_{i} \cdot \alpha_{j}=\beta_{i} \cdot \beta_{j}=0$, $\alpha_{i} \cdot \beta_{j}=\delta_{i j}$.

Define the period matrices by

$$
\left[\omega^{\prime} \omega^{\prime \prime}\right]=\left[\int_{\alpha_{i}} \omega_{j} \int_{\beta_{i}} \omega_{j}\right]_{i, j=1,2,3},\left[\eta^{\prime} \eta^{\prime \prime}\right]=\left[\int_{\alpha_{i}} \eta_{j} \int_{\beta_{i}} \eta_{j}\right]_{i, j=1,2,3} .
$$


We can combine these two matrices into

$$
M=\left[\begin{array}{ll}
\omega^{\prime} & \omega^{\prime \prime} \\
\eta^{\prime} & \eta^{\prime \prime}
\end{array}\right]
$$

Then $M$ satisfies

$$
M\left[\begin{array}{ll} 
& -1_{3} \\
1_{3} &
\end{array}\right]{ }^{t} M=2 \pi \sqrt{-1}\left[\begin{array}{ll} 
& -1_{3} \\
1_{3} &
\end{array}\right]
$$

This is the generalized Legendre relation (see (1.14) on p. 11 of [9]). In particular, $\omega^{\prime-1} \omega^{\prime \prime}$ is a symmetric matrix. We know also that

$$
\operatorname{Im}\left(\omega^{\prime-1} \omega^{\prime \prime}\right) \quad \text { is positive definite. }
$$

By looking at (2.2), we see the canonical divisor class of $C$ is given by $4 \infty$ and we are taking $\infty$ as the base point of the Abel map (2.6) the Riemann constant is an element of $\left(\frac{1}{2} \mathbb{Z}\right)^{6}$ (see [22], Corollary 3.11, p. 166). Let

$$
\delta:=\left[\begin{array}{l}
\delta^{\prime} \\
\delta^{\prime \prime}
\end{array}\right] \in\left(\frac{1}{2} \mathbb{Z}\right)^{6}
$$

be the theta characteristic which gives the Riemann constant with respect to the base point $\infty$ and to the period matrix $\left[\omega^{\prime} \omega^{\prime \prime}\right]$. Note that we use $\delta^{\prime}, \delta^{\prime \prime}$ as well as $n$ in (4.8) as columns, to keep the notation a bit simpler. We define

$$
\begin{aligned}
\sigma(u)= & \sigma(u ; M)=\sigma\left(u_{1}, u_{2}, u_{3} ; M\right) \\
= & c \exp \left(-\frac{1}{2} u \eta^{\prime} \omega^{\prime-1} t u\right) \vartheta[\delta]\left(\omega^{\prime-1} t u ; \omega^{\prime-1} \omega^{\prime \prime}\right) \\
= & c \exp \left(-\frac{1}{2} u \eta^{\prime} \omega^{\prime-1} t u\right) \sum_{n \in \mathbb{Z}^{3}} \\
& \times \exp \left[2 \pi i\left\{\frac{1}{2}^{t}\left(n+\delta^{\prime}\right) \omega^{\prime-1} \omega^{\prime \prime}\left(n+\delta^{\prime}\right)+{ }^{t}\left(n+\delta^{\prime}\right)\left(\omega^{\prime-1} t u+\delta^{\prime \prime}\right)\right\}\right],
\end{aligned}
$$

where

$$
C=\frac{1}{\sqrt[8]{D}}\left(\frac{\pi^{3}}{\left|\omega^{\prime}\right|}\right)^{1 / 2}
$$

with $D$ from (2.14). Here the choice of a root of (4.9) is explained in the Remark 7.2 below. The series (4.8) converges because of (4.6). 
In what follows, for a given $u \in \mathbb{C}^{3}$, we denote by $u^{\prime}$ and $u^{\prime \prime}$ the unique elements in $\mathbb{R}^{3}$ such that

$$
u=u^{\prime} \omega^{\prime}+u^{\prime \prime} \omega^{\prime \prime}
$$

Then for $u, v \in \mathbb{C}^{3}$, and $\ell\left(=\ell^{\prime} \omega^{\prime}+\ell^{\prime \prime} \omega^{\prime \prime}\right) \in \Lambda$, we define

$$
\begin{aligned}
L(u, v) & :=u\left(\eta^{\prime t} v^{\prime}+\eta^{\prime \prime} v^{\prime \prime}\right), \\
\chi(\ell) & :=\exp \left[\pi \sqrt{-1}\left(2\left(\ell^{\prime} \delta^{\prime \prime}-\ell^{\prime \prime} \delta^{\prime}\right)+\ell^{\prime t} \ell^{\prime \prime}\right)\right](\in\{1,-1\}) .
\end{aligned}
$$

In this situation the most important properties of $\sigma(u ; M)$ are as follows:

LEMMA 4.1. The function $\sigma(u)$ is an entire function. For all $u \in \mathbb{C}^{3}, \ell \in \wedge$ and $\gamma \in$ $\operatorname{Sp}(6, \mathbb{Z})$, we have

$$
\begin{aligned}
\sigma(u+\ell ; M) & =\chi(\ell) \sigma(u ; M) \exp L\left(u+\frac{1}{2} \ell, \ell\right), \\
\sigma(u ; \gamma M) & =\sigma(u ; M), \\
u \mapsto \sigma(u ; M) & \text { has zeroes of order } 1 \text { along } \Theta^{[2]}, \\
\sigma(u ; M) & =0 \Longleftrightarrow u \in \Theta^{[2]} .
\end{aligned}
$$

PROOF. The function $\sigma$ is clearly entire from its definition and from the known property of theta series. The formula (4.12) is a special case of the equation from [1] (p. 286 in the 1995 reprint, $\ell .22)$. The statement (4.13) is easily shown by using the definition of $\sigma(u)$ since $\gamma$ corresponds to changing the choice of the paths of integration given in (4.3). The statements (4.14) and (4.15) are explained in [1], (p. 252). These facts are partially described also in [9], (p. 12, Theorem 1.1 and p. 15).

LEMMA 4.2. The function $\sigma(u)$ is either odd or even, i.e.

$$
\sigma([-1] u)=-\sigma(u) \quad \text { or } \quad \sigma([-1] u)=\sigma(u) .
$$

PRoOF. We fix a matrix $M$ satisfying (4.5) and (4.6). Therefore the bilinear form $L($, ) is fixed. Then the space of the solutions of (4.12) is one dimensional over $\mathbb{C}$, because the Pfaffian of the Riemann form attached to $L($, ) is 1 (see [24], Lemma 3.1.2 and [20], p. 93, Theorem 3.1). Hence, such nontrivial solutions automatically satisfy (4.13) and (4.15); while (4.14) requires the constant factor to be the same, this is guaranteed 
by the definition of $\sigma$ and the fact that (4.9) is independent of $\gamma$. In this sense, (4.12) characterizes the function $\sigma(u)$ up to a constant, which depends only on the $\mu_{j}$ s. Now considering the loop integrals for $\omega$ in the reverse direction, we see that

$$
[-1] \wedge=\wedge
$$

Hence $u \mapsto \sigma([-1] u)$ satisfies (4.12) also. So there exists a constant $K$ such that

$$
\sigma([-1] u)=K \sigma(u)
$$

Since $[-1]^{2}$ is trivial, it must be $K^{2}=1$.

REMARK 4.3. In fact $\sigma(u)$ is an odd function as we see in the Theorem 7.1.

We need the power series expansion of $\sigma(u)$ with respect to $u_{1}, u_{2}, u_{3}$. To get the expansion, first of all, we need to investigate Abelian functions given by logarithmic (higher) derivatives of $\sigma(u)$. We shall examine this in the next Section.

\section{Standard Abelian Functions}

DEFINITION 5.1. A meromorphic function $u \mapsto \mathfrak{P}(u)$ on $\mathbb{C}^{3}$ is called a standard Abelian function if it is holomorphic outside $\kappa^{-1}\left(\Theta^{[2]}\right)$ and is multi-periodic, namely, if it satisfies

$$
\mathfrak{P}\left(u+\omega^{\prime} n+\omega^{\prime \prime} m\right)=\mathfrak{P}(u)
$$

for all integer vectors $n, m \in \mathbb{Z}$ and all $u \notin \kappa^{-1}\left(\Theta^{[2]}\right)$.

To realize the standard Abelian functions in terms of the $\sigma$-function, we first let

$$
\Delta_{i}=\frac{\partial}{\partial u_{i}}-\frac{\partial}{\partial v_{i}}
$$

for $u=\left(u_{1}, u_{2}, u_{3}\right)$ and $v=\left(v_{1}, v_{2}, v_{3}\right)$. This operator occurs in what is now known as Hirota's bilinear operator, but in fact was introduced much earlier in the PDE case by Baker ([3], p. 151, [4], p. 49) (see also [14]). We define fundamental Abelian functions on $J$ by

$$
\wp_{i j}(u)=-\left.\frac{1}{2 \sigma(u)^{2}} \Delta_{i} \Delta_{j} \sigma(u) \sigma(v)\right|_{V=u}=-\frac{\partial^{2}}{\partial u_{i} \partial u_{j}} \log \sigma(u)
$$


It follows from (4.16) that these functions are even. For the benefit of the reader familiar with the genus one case, we should point out that the Weierstrass function $\wp(u)$ described in Equation (1.1) would be written as $\wp_{11}(u)$ in this notation. It is clear that they belong to $\Gamma\left(J, 2 \Theta^{[2]}\right)$. Moreover, we define

$$
\wp_{i j k}(u)=\frac{\partial}{\partial u_{k}} \wp_{i j}(u), \quad \wp_{i j k \ell}(u)=\frac{\partial}{\partial u_{\ell}} \wp_{i j k}(u) .
$$

The 3-index $\wp$-functions are odd and 4-index $\wp$ are even by Lemma 4.2. The functions (5.3) and (5.4) are standard Abelian functions from Lemma 4.1. Following (and generalizing) Baker ([3], p. 151, [4], pp. 49-50) (see also [10], pp. 18-19, or [13]), we define

$$
\begin{aligned}
Q_{i j k \ell}(u) & =-\left.\frac{1}{2 \sigma(u)^{2}} \Delta_{i} \Delta_{j} \Delta_{k} \Delta_{\ell} \sigma(u) \sigma(v)\right|_{V=u} \\
& =\wp_{i j k \ell}(u)-2\left(\wp_{i j} \wp_{k \ell}+\wp_{i k} \wp_{j \ell}+\wp_{i \ell} \wp_{j k}\right)(u),
\end{aligned}
$$

which specializes to

$$
\begin{aligned}
& Q_{i j k k}=\wp_{i j k k}-2 \wp_{i j} \wp_{k k}-4 \wp_{i k} \wp_{j k}, \quad \quad Q_{i i k k}=\wp_{i i k k}-2 \wp_{i i} \wp_{k k}-4 \wp_{i k}^{2} \text {, } \\
& Q_{i k k k}=\wp_{i k k k}-6 \wp_{i k} \wp_{k k}, \quad \quad Q_{k k k k}=\wp_{k k k k}-6 \wp_{k k}{ }^{2} .
\end{aligned}
$$

A short calculation shows that $Q_{i j k \ell}$ belongs in $\Gamma\left(J, \mathcal{O}\left(2 \Theta^{[2]}\right)\right)$, whereas $\wp_{i j k \ell}$ belongs in $\Gamma\left(J, \mathcal{O}\left(4 \Theta^{[2]}\right)\right)$. In particular $Q_{1333}$ plays a key role in what follows.

Note that although the subscripts in $\wp_{i j k \ell}$ do denote differentiation, the subscripts in $Q_{i j k \ell}$ do not denote direct differentiation, and the latter notation is introduced for convenience only. This is important to bear in mind when we use crossdifferentiation, for example the $\wp_{i j k \ell}$ satisfy

$$
\frac{\partial}{\partial u_{m}} \wp_{i j k \ell}(u)=\frac{\partial}{\partial u_{\ell}} \wp_{i j k m}(u),
$$

whereas the $Q_{i j k \ell}$ do not. The following useful formula (5.6) involving fundamental Kleinian functions, for the case of the general curve (2.1), was derived in [10]. It would be helpful for the reader to see [2], p. 377 for the case of hyperelliptic curves. The formula (5.6) below is proved similarly.

PROPOSITION 5.2. Let $u \in \mathbb{C}^{3}$ and $\left(x_{1}, y_{1}\right),\left(x_{2}, y_{2}\right),\left(x_{3}, y_{3}\right)$ be Abelian preimages of u, i.e.

$$
u=\int_{\infty}^{\left(x_{1}, Y_{1}\right)} \omega+\int_{\infty}^{\left(x_{2}, Y_{2}\right)} \omega+\int_{\infty}^{\left(x_{3}, Y_{3}\right)} \omega
$$


with appropriate paths of the integrals. Let $(x, y)$ be an arbitrary point on the curve $C$. Then, for each $k=1,2,3$, the following formula holds

$$
\left[\begin{array}{lll}
1 & x & y
\end{array}\right]\left[\wp_{i j}\left(\int_{\infty}^{(x, y)} \omega-u\right)\right]\left[\begin{array}{c}
1 \\
x_{k} \\
y_{k}
\end{array}\right]=\frac{F\left((x, y),\left(x_{k}, y_{k}\right)\right)}{\left(x-x_{k}\right)^{2}}
$$

where $F((x, y),(z, w))$ is a polynomial defined by (2.17) or (A.3).

PROOF. Using (4.14) and relations of differentials of the second kind on $C$ with ones of the third kind (see $[1]$, p. $22, \ell .15$ and p. 22, $\ell .11$ ), we have an equation connecting the theta series in (4.8) and differentials of the third kind (see [1], p. 275, Ł.-11, for example). Then this equation is modified into a form which involves $\sigma(u)$ and the 2 -form $\Omega((x, y),(z, w))$ of (2.19). Finally, after taking logarithm of the modified one, applying $\frac{\partial^{2}}{\partial u_{i} \partial u_{j}}$ to it gives the desired equation.

PROPOSITION 5.3. Suppose the $\left(x_{i}, y_{i}\right)$ s and $u$ are related as in Proposition 5.2. Let $(x, y)$ be any one of $\left(x_{i}, y_{i}\right)$ s. Then we have infinitely many relations, of homogeneous weight, linear in

$$
\wp_{i j}(u), \wp_{i j k}(u), \wp_{i j k \ell}(u), \cdots \quad(i, j, k=1,2,3),
$$

and whose coefficients are polynomials of $x, y$ and $\mu_{j}$ s. We list the first three of them of lower weights as follows:

$$
\begin{aligned}
& \wp_{33}(u) y+\wp_{23}(u) x+\wp_{13}(u)=x^{2}, \\
& \left(\wp_{23}(u)+\frac{1}{3} \mu_{1} \wp_{33}(u)-\wp_{333}(u)\right) y+\left(\wp_{22}(u)-\wp_{233}(u)\right. \\
& \left.\quad+\frac{1}{3} \mu_{1} \wp_{23}(u)\right) x+\frac{1}{3} \mu_{1} \wp_{13}(u)+\wp_{12}(u)-\wp_{133}(u)=2 x y-\frac{2}{3} \mu_{1} x^{2}, \\
& -3 y^{2}+\left(\frac{1}{3} \wp_{33} \mu_{2}+\frac{1}{2} \wp_{3333}-\frac{1}{2} \mu_{1} \wp_{333}+\frac{1}{9} \mu_{1}^{2} \wp_{33}+2 \mu_{1} x-\frac{3}{2} \wp_{233}+2 \mu_{4}\right) y \\
& \quad+\left(\frac{2}{3} \mu_{2}-\frac{1}{9} \mu_{1}^{2}\right) x^{2}+\left(-\frac{1}{2} \mu_{1} \wp_{233}+\mu_{5}+\frac{1}{2} \wp_{2333}+\frac{1}{3} \wp_{23} \mu_{2}+\frac{1}{9} \mu_{1}^{2} \wp_{23}-\frac{3}{2} \wp_{223}\right) x \\
& \quad+\frac{1}{2} \wp_{1333}+\frac{1}{3} \mu_{2} \wp_{13}+\mu_{8}+\frac{1}{9} \mu_{1}^{2} \wp_{13}-\frac{3}{2} \wp_{123}-\frac{1}{2} \mu_{1} \wp_{133}=0 .
\end{aligned}
$$

More equations of this type are available in [15].

PROOF. These relations are derived from (5.6) by expanding it with respect to a local parameter $t=x^{-1 / 3}$, in the vicinity of the point at infinity, and comparing the principal parts of the poles on both sides of the relation (5.6), we find the solution of the Jacobi inversion problem. 
REMARK 5.4.

(1) In the case of trigonal curves, a formula of this type was first given explicitly for a particular case of the curve (2.1) in [16].

(2) We use in the proof of Lemma 6.1 below the first seven relations in Proposition (5.3). Namely, those of weight from -6 to -12 .

The first two relations in 5.3 give solution of the Jacobi inversion problem (see also [10]):

COROLLARY 5.5. Suppose the $\left(x_{i}, y_{i}\right) s$ and $u$ are related as in Proposition 5.2. The solution of the Jacobi inversion problem is given by $\left(x_{1}, y_{1}\right),\left(x_{2}, y_{2}\right)$, and $\left(x_{3}, y_{3}\right)$, where these points are the set of zeros of the equations (5.7), (5.8) for $(x, y)$.

We remark that the right hand sides of equations (5.7), (5.8) are related to the polynomials $h_{3}(z, w)$ and $h_{2}(z, w)$ defining the canonical meromorphic differentials $\eta_{3}(z, w)$ and $\eta_{2}(z, w)$. Further, the equation (5.8) is directly related to the determinant of the matrices constructed in [25], using the algebraic approach developed in [21].

If we take the resultant of (5.7), (5.8) with respect to $y$, we find a cubic equation in $x$ which can be used to substitute for $x^{3}$ in terms of lower powers of $x$

$$
\begin{aligned}
x^{3}=\frac{1}{2} & \left(3 \wp_{23}+\mu_{1} \wp_{33}-\wp_{333}\right) x^{2}+\frac{1}{2}\left(\wp_{33} \wp_{22}+2 \wp_{13}+\wp_{23} \wp_{333}-\wp_{33} \wp_{233}-\wp_{23}{ }^{2}\right) x \\
& +\frac{1}{2} \wp_{33} \wp_{12}-\frac{1}{2} \wp_{33} \wp_{133}-\frac{1}{2} \wp_{13} \wp_{23}+\frac{1}{2} \wp_{13} \wp_{333} .
\end{aligned}
$$

If we now take the resultant of (5.7), (5.9) with respect to $y$, we get a quartic in $x$ which can be reduced to a quadratic by repeated use of (5.10). This quadratic in $x$ is not further reducible. A quadratic equation in $x$ has at most only two solutions and $u$ has three free variables. Hence each of the coefficients of $1, x, x^{2}$ of the quadratic must be identically zero. Furthermore, each coefficient can be split into two parts which are even and odd under the reflection (2.7), and each of these parts must vanish. So each term of order higher than two in the expansion of (5.6) will give up to six separate equations involving the $\wp$ functions. The simplest two arising from the resultant of (5.7), (5.9) are

$$
\begin{array}{r}
\wp_{222}-2 \wp_{33} \wp_{233}+2 \wp_{23} \wp_{333}-\mu_{2} \wp_{233}+\mu_{3} \wp_{333}+\mu_{1} \wp_{223}=0 \\
\wp_{23} \wp_{233}-2 \wp_{33} \wp_{223}+\wp_{333} \wp_{22}+2 \wp_{133}+\mu_{1}\left(\wp_{23} \wp_{333}-\wp_{33} \wp_{233}\right)=0
\end{array}
$$

where $\wp_{i j}=\wp_{i j}(u)$ and $\wp_{i j k}=\wp_{i j k}(u)$. 


\section{Equations Satisfied by the Abelian Functions for the General Trigonal Case}

We can use the expansion of (5.6) as described in the discussion following Corollary 5.5 to derive various equations which the Abelian functions defined by (5.4) and (5.5) must satisfy. We consider first the 4-index equations, the generalizations of $\wp^{\prime \prime}=6 \wp^{2}-\frac{1}{2} g_{2}$ in the cubic (genus 1) case.

LEMMA 6.1. The 4-index functions $\wp_{i j k \ell}$ associated with (3.1) satisfy the following relations:

$$
\begin{aligned}
& \wp_{3333}=6 \wp_{33}^{2}+\mu_{1}^{2} \wp_{33}-3 \wp_{22}+2 \mu_{1} \wp_{23}-4 \mu_{2} \wp_{33}-2 \mu_{4}, \\
& \wp_{2333}=6 \wp_{23} \wp_{33}+\mu_{1}^{2} \wp_{23}+3 \mu_{3} \wp_{33}-\mu_{2} \wp_{23}-\mu_{5}-\mu_{1} \wp_{22} \text {, } \\
& \wp_{2233}=4 \wp_{23}^{2}+2 \wp_{33} \wp_{22}+\mu_{1} \mu_{3} \wp_{33}-\mu_{2} \wp_{22}+2 \mu_{6}+3 \mu_{3} \wp_{23}+\mu_{1} \mu_{2} \wp_{23}+4 \wp_{13}, \\
& \wp_{2223}=6 \wp_{22} \wp_{23}+4 \mu_{1} \wp_{13}+\mu_{1} \mu_{3} \wp_{23}+\mu_{2} \mu_{3} \wp_{33}+2 \mu_{3} \mu_{4}+\mu_{2}{ }^{2} \wp_{23}+4 \mu_{4} \wp_{23}+3 \mu_{3} \wp_{22} \\
& +2 \mu_{1} \mu_{6}+\mu_{2} \mu_{5}-2 \mu_{5} \wp_{33} \\
& \wp_{2222}=6 \wp_{22}^{2}-2 \mu_{2} \mu_{3} \wp_{23}+\mu_{1} \mu_{2} \mu_{5}+2 \mu_{1} \mu_{3} \mu_{4}+24 \wp_{13} \wp_{33}+4 \mu_{1}^{2} \wp_{13}-4 \mu_{2} \wp_{13} \\
& -4 \wp_{1333}+4 \mu_{5} \wp_{23}+2 \mu_{1}^{2} \mu_{6}-2 \mu_{2} \mu_{6}+\mu_{3} \mu_{5}-3 \mu_{3}^{2} \wp_{33}+12 \mu_{6} \wp_{33}+4 \mu_{4} \wp_{22} \\
& +\mu_{2}^{2} \wp_{22}+4 \mu_{1} \mu_{3} \wp_{22} \\
& \wp_{1233}=4 \wp_{13} \wp_{23}+2 \wp_{33} \wp_{12}-2 \mu_{1} \wp_{33} \wp_{13}-\frac{1}{3} \mu_{1}^{3} \wp_{13}+\frac{1}{3} \mu_{1} \wp_{1333}+\frac{1}{3} \mu_{1}^{2} \wp_{12}+3 \mu_{3} \wp_{13} \\
& +\frac{1}{3} \mu_{1} \mu_{8}+\frac{4}{3} \mu_{1} \mu_{2} \wp_{13}-\mu_{2} \wp_{12}+\mu_{9} \\
& \wp_{1223}=4 \wp_{23} \wp_{12}+2 \wp_{13} \wp_{22}-2 \mu_{2} \wp_{33} \wp_{13}-2 \mu_{8} \wp_{33}-\frac{2}{3} \mu_{8} \mu_{2}+\frac{1}{3} \mu_{2} \wp_{1333}+3 \mu_{3} \wp_{12} \\
& +4 \mu_{4} \wp_{13}+\frac{4}{3} \mu_{2}^{2} \wp_{13}-2 \wp_{11}-\frac{1}{3} \mu_{1}^{2} \mu_{2} \wp_{13}+\frac{1}{3} \mu_{1} \mu_{2} \wp_{12}+\mu_{1} \mu_{3} \wp_{13}, \\
& \wp_{1222}=6 \wp_{22} \wp_{12}+6 \mu_{9} \wp_{33}-\mu_{3} \wp_{1333}+4 \mu_{5} \wp_{13}+\mu_{2}^{2} \wp_{12}-\mu_{2} \mu_{9}+4 \mu_{4} \wp_{12}-2 \mu_{1} \wp_{11} \\
& +6 \mu_{3} \wp_{33} \wp_{13}-3 \mu_{2} \mu_{3} \wp_{13}+\mu_{1}^{2} \mu_{3} \wp_{13}+3 \mu_{1} \mu_{3} \wp_{12}-\mu_{1} \mu_{2} \mu_{8}, \\
& \wp_{1133}=4 \wp_{13}^{2}+2 \wp_{33} \wp_{11}-\mu_{9} \wp_{23}+2 \mu_{6} \wp_{13}+\mu_{8} \wp_{22}-\mu_{5} \wp_{12}+\frac{2}{3} \mu_{4} \wp_{1333}+\frac{2}{3} \mu_{4} \mu_{8} \\
& +2 \mu_{2} \mu_{8} \wp_{33}-4 \mu_{4} \wp_{13} \wp_{33}+\frac{2}{3} \mu_{2} \mu_{4} \wp_{13}+\mu_{1} \mu_{9} \wp_{33}-\mu_{1} \mu_{8} \wp_{23}+\mu_{1} \mu_{5} \wp_{13} \\
& -\frac{2}{3} \mu_{1}^{2} \mu_{4} \wp_{13}+\frac{2}{3} \mu_{1} \mu_{4} \wp_{12} \\
& \wp_{1123}=4 \wp_{12} \wp_{13}+2 \wp_{23} \wp_{11}+2 \mu_{3} \mu_{4} \wp_{13}-\mu_{3} \mu_{8} \wp_{33}-2 \mu_{5} \wp_{13} \wp_{33}+\mu_{2} \mu_{8} \wp_{23}+\frac{4}{3} \mu_{2} \mu_{5} \wp_{13} \\
& -\mu_{9} \wp_{22}+2 \mu_{6} \wp_{12}+\frac{1}{3} \mu_{5} \wp_{1333}+\frac{1}{3} \mu_{5} \mu_{8}+\mu_{1} \mu_{9} \wp_{23}-\frac{1}{3} \mu_{1}^{2} \mu_{5} \wp_{13}+\frac{1}{3} \mu_{1} \mu_{5} \wp_{12}, \\
& \wp_{1122}=4 \wp_{12}^{2}+2 \wp_{11} \wp_{22}+\frac{2}{3} \mu_{1}^{2} \mu_{6} \wp_{13}+\frac{4}{3} \mu_{1} \mu_{6} \wp_{12}+\mu_{3} \mu_{9} \wp_{33}+\mu_{2} \mu_{9} \wp_{23}+8 \mu_{12} \wp_{33} \\
& +2 \mu_{3} \mu_{4} \wp_{12}-\frac{2}{3} \mu_{6} \wp_{1333}+4 \mu_{8} \wp_{13}-\frac{2}{3} \mu_{6} \mu_{8}+4 \mu_{6} \wp_{33} \wp_{13}-\mu_{3} \mu_{8} \wp_{23}+\mu_{3} \mu_{5} \wp_{13}
\end{aligned}
$$




$$
\begin{aligned}
& -\frac{8}{3} \mu_{2} \mu_{6} \wp_{13}+\mu_{2} \mu_{8} \wp_{22}+\mu_{2} \mu_{5} \wp_{12} \\
& \wp_{1113}=6 \wp_{13} \wp_{11}+6 \mu_{2} \mu_{8} \wp_{13}-2 \mu_{2} \mu_{12} \wp_{33}-\mu_{1}^{2} \mu_{8} \wp_{13}+4 \mu_{1} \mu_{12} \wp_{23}+\mu_{1} \mu_{8} \wp_{12}+\mu_{5} \mu_{9} \wp_{33} \\
& +\mu_{5}{ }^{2} \wp_{13}-2 \mu_{4} \mu_{9} \wp_{23}+\mu_{1} \mu_{9} \wp_{13}-6 \mu_{8} \wp_{33} \wp_{13}-2 \mu_{6} \mu_{8} \wp_{33}+\mu_{8} \wp_{1333}-4 \mu_{4} \mu_{12} \\
& +3 \mu_{9} \wp_{12}-6 \mu_{12} \wp_{22}-\mu_{5} \mu_{8} \wp_{23}+4 \mu_{4} \mu_{6} \wp_{13} \\
& \wp_{1112}=6 \wp_{12} \wp_{11}+6 \mu_{3} \mu_{12} \wp_{33}+3 \mu_{3} \mu_{8} \wp_{13}-2 \mu_{6} \mu_{8} \wp_{23}-\mu_{1} \mu_{8}^{2}+5 \mu_{2} \mu_{8} \wp_{12}+4 \mu_{2} \mu_{12} \wp_{23} \\
& -2 \mu_{1} \mu_{12} \wp_{22}+4 \mu_{4} \mu_{6} \wp_{12}-\mu_{5} \mu_{8} \wp_{22}+\mu_{5}^{2} \wp_{12}+4 \mu_{5} \mu_{12}-\mu_{9} \wp_{1333}-4 \mu_{1} \mu_{12} \mu_{4} \\
& +\mu_{1}^{2} \mu_{9} \wp_{13}+3 \mu_{1} \mu_{9} \wp_{12}-2 \mu_{4} \mu_{9} \wp_{22}+\mu_{5} \mu_{9} \wp_{23}-4 \mu_{2} \mu_{9} \wp_{13} \\
& +6 \mu_{9} \wp_{13} \wp_{33}-3 \mu_{8} \mu_{9} \\
& \wp_{1111}=6 \wp_{11}{ }^{2}+4 \mu_{4} \mu_{9} \wp_{12}-8 \mu_{4}{ }^{2} \mu_{12}-2 \mu_{2}{ }^{2} \mu_{4} \mu_{12}-3 \mu_{8}{ }^{2} \wp_{22}-2 \mu_{4} \mu_{8}{ }^{2}+\mu_{5}{ }^{2} \wp_{11} \\
& -3 \mu_{9}^{2} \wp_{33}-4 \mu_{12} \wp_{1333}+24 \mu_{12} \wp_{33} \wp_{13}+12 \mu_{5} \mu_{12} \wp_{23}+\mu_{2} \mu_{4} \mu_{5} \mu_{9}-6 \mu_{1} \mu_{3} \mu_{4} \mu_{12} \\
& +\mu_{1} \mu_{2} \mu_{5} \mu_{12}+2 \mu_{6}{ }^{2} \mu_{8}+2 \mu_{2}{ }^{2} \mu_{8}{ }^{2}-\mu_{5} \mu_{6} \mu_{9}-2 \mu_{5} \mu_{9} \wp_{13}+4 \mu_{4} \mu_{6} \wp_{11}+4 \mu_{6} \mu_{8} \wp_{13} \\
& +8 \mu_{2} \mu_{8} \wp_{11}-6 \mu_{2} \mu_{6} \mu_{12}-12 \mu_{2} \mu_{12} \wp_{13}+4 \mu_{1}{ }^{2} \mu_{12} \wp_{13}+2 \mu_{1}{ }^{2} \mu_{6} \mu_{12}+2 \mu_{8} \mu_{5} \wp_{12} \\
& -6 \mu_{8} \mu_{9} \wp_{23}-12 \mu_{4} \mu_{12} \wp_{22}+\mu_{2} \mu_{5}{ }^{2} \mu_{8}+2 \mu_{1} \mu_{4} \mu_{6} \mu_{9}+\mu_{1} \mu_{5} \mu_{6} \mu_{8}+12 \mu_{6} \mu_{12} \wp_{33} \\
& +4 \mu_{1} \mu_{9} \wp_{11}+2 \mu_{3} \mu_{4}^{2} \mu_{9}+9 \mu_{3} \mu_{5} \mu_{12}-2 \mu_{1} \mu_{3} \mu_{8}^{2}-6 \mu_{3} \mu_{8} \mu_{9}+2 \mu_{1} \mu_{2} \mu_{8} \mu_{9} \\
& +\mu_{3} \mu_{4} \mu_{5} \mu_{8}+2 \mu_{2} \mu_{4} \mu_{6} \mu_{8}+2 \mu_{2} \mu_{9}{ }^{2} \text {. }
\end{aligned}
$$

PROOF. Many of these relations follow from the sets of equations generated from the first seven terms of the expansion of (5.6) as indicated in Proposition 5.3 by a similar argument as that explained at the end of the previous Section. Others can be derived making use of derivatives of the equations in Lemma 6.5, or products of these equations with 3 -index expressions $\wp_{i j k}$, working in a self-consistent way from higher to lower weights. The calculations are somewhat long and tedious and much facilitated by heavy use of Maple. Full Maple worksheets are available on request from the authors.

REMARK 6.2. The complete set of the 4-index relations for $\wp$-functions for genus three was derived by Baker [3] in the hyperelliptic case only. As far as we know, the above relations are new, and a comparison with Baker's relations is of interest.

REMARK 6.3. With the use of (5.5), these equations can be written in a slightly more compact form involving the $Q_{i j k \ell}$ functions. For example, the sixth equation (for $\wp_{2222}$ ) becomes

$$
\begin{gathered}
O_{2222}=-2 \mu_{2} \mu_{3} \wp_{23}+\mu_{1} \mu_{2} \mu_{5}+2 \mu_{1} \mu_{3} \mu_{4}+4 \mu_{1}^{2} \wp_{13}-4 \mu_{2} \wp_{13}-4 Q_{1333}+4 \mu_{5} \wp_{23} \\
+2 \mu_{1}^{2} \mu_{6}-2 \mu_{2} \mu_{6}+\mu_{3} \mu_{5}-3 \mu_{3}^{2} \wp_{33}+12 \mu_{6} \wp_{33}+4 \mu_{4} \wp_{22}+\mu_{2}^{2} \wp_{22}+4 \mu_{1} \mu_{3} \wp_{22} .
\end{gathered}
$$


J. C. Eilbeck et al.

The importance of this switch to the $Q$ variables is that the equations become linear in the $Q_{i j k \ell}$ and the 2-index $\wp_{i j}$. An alternative way of looking at this is that the equations in Lemma 6.1 have only second-order poles in $\sigma$.

REMARK 6.4. The first relation in Lemma 6.1, after differentiating twice with respect to $u_{3}$, becomes the Boussinesq equation for the function $\wp_{33}($ see $[10,16])$.

LEMMA 6.5. The 3-index functions $\wp_{i j k}$ associated with (3.1) satisfy a number of bilinear relations (linear in both 3-index and 2-index functions). These have no analog in the genus 1 case. For example, in decreasing weight, starting at -6 we have

$$
\begin{aligned}
& -2 \wp_{33} \wp_{233}+2 \wp_{23} \wp_{333}+\mu_{3} \wp_{333}+\mu_{2} \wp_{233}-\mu_{1} \wp_{223}+\wp_{222}=0, \quad[-6] \\
& -2 \wp_{33} \wp_{223}+\mu_{1} \wp_{33} \wp_{233}+\wp_{23} \wp_{233}-\mu_{1} \wp_{23} \wp_{333}+\wp_{333} \wp_{22}+2 \wp_{133}=0, \quad[-7] \\
& -2 \wp_{23} \wp_{223}+4 \wp_{22} \wp_{233}+4 \mu_{1} \wp_{133}+\mu_{3} \mu_{2} \wp_{333}+\mu_{2}^{2} \wp_{233} \\
& +4 \mu_{4} \wp_{233}-2 \mu_{5} \wp_{333}-2 \wp_{33} \wp_{222}+\mu_{2} \wp_{222}-\mu_{1} \mu_{2} \wp_{223}-4 \wp_{123}=0, \quad[-8] \\
& 3 \mu_{1} \mu_{3} \wp_{223}-3 \mu_{2} \mu_{3} \wp_{233}-24 \wp_{33} \wp_{133}+24 \wp_{13} \wp_{333}+12 \wp_{122}-12 \mu_{1} \wp_{123} \\
& +12 \mu_{2} \wp_{133}-3 \mu_{3} \wp_{222}+6 \mu_{5} \wp_{233}-3 \mu_{3}^{2} \wp_{333}+12 \mu_{6} \wp_{333}-6 \wp_{23} \wp_{222} \\
& +6 \wp_{22} \wp_{223}=0, \quad[-9] \\
& 2 \wp_{33} \wp_{123}-\mu_{1} \wp_{33} \wp_{133}+\mu_{1} \wp_{13} \wp_{333}+\wp_{23} \wp_{133}-\wp_{12} \wp_{333}-2 \wp_{13} \wp_{233}=0, \quad[-10] \\
& \wp_{113}+\wp_{13} \wp_{223}-2 \mu_{4} \wp_{133}+\wp_{33} \wp_{122}-\wp_{22} \wp_{133}-\wp_{12} \wp_{233}+\mu_{8} \wp_{333}-\mu_{2} \wp_{133} \wp_{33} \\
& -\mu_{1} \wp_{13} \wp_{233}+\mu_{2} \wp_{13} \wp_{333}+\mu_{1} \wp_{133} \wp_{23}=0, \quad[-11] \\
& -\wp_{112}-3 \mu_{9} \wp_{333}+\wp_{13} \wp_{222}-\wp_{12} \wp_{223}-2 \wp_{22} \wp_{123}-2 \mu_{5} \wp_{133}+\mu_{1} \wp_{113}+2 \wp_{23} \wp_{122} \\
& -\mu_{8} \wp_{233}-\mu_{2} \wp_{13} \wp_{233}+3 \mu_{3} \wp_{33} \wp_{133}-3 \mu_{3} \wp_{13} \wp_{333}+\mu_{2} \wp_{23} \wp_{133}=0, \quad[-12] \\
& 8 \mu_{4} \wp_{133} \wp_{33}-8 \mu_{4} \wp_{13} \wp_{333}-4 \mu_{2} \mu_{4} \wp_{133}+2 \mu_{1} \mu_{9} \wp_{333}-2 \mu_{1} \mu_{8} \wp_{233}+2 \mu_{1} \mu_{5} \wp_{133} \\
& +4 \mu_{1} \mu_{4} \wp_{123}+4 \mu_{8} \mu_{2} \wp_{333}+3 \mu_{3} \wp_{13} \wp_{233}-3 \mu_{3} \wp_{23} \wp_{133}-\mu_{1} \wp_{112}+3 \wp_{12} \wp_{222} \\
& +4 \wp_{11} \wp_{333}-2 \mu_{6} \wp_{133}-3 \wp_{122} \wp_{22}-4 \mu_{4} \wp_{122}+\mu_{9} \wp_{233}+2 \mu_{8} \wp_{223}-8 \wp_{33} \wp_{113} \\
& +4 \wp_{13} \wp_{133}-2 \mu_{1}^{2} \wp_{113}+2 \mu_{2} \wp_{113}-2 \mu_{5} \wp_{123}=0, \quad[-13] \\
& 4 \wp_{123} \wp_{13}+4 \mu_{4} \wp_{23} \wp_{133}+\mu_{3} \mu_{8} \wp_{333}-2 \mu_{5} \wp_{33} \wp_{133}+2 \mu_{5} \wp_{13} \wp_{333}+\mu_{2} \mu_{8} \wp_{233}+\mu_{8} \wp_{222} \\
& -4 \wp_{12} \wp_{133}-2 \wp_{23} \wp_{113}+2 \wp_{33} \wp_{112}-4 \mu_{4} \wp_{13} \wp_{233}-\mu_{1} \mu_{8} \wp_{223}=0, \quad[-14] \\
& -\mu_{9} \wp_{222}+\mu_{1} \mu_{9} \wp_{223}+4 \wp_{13} \wp_{122}+2 \wp_{23} \wp_{112}-2 \wp_{113} \wp_{22}-\mu_{3} \mu_{9} \wp_{333}-\mu_{2} \mu_{9} \wp_{233} \\
& +2 \mu_{5} \wp_{23} \wp_{133}-8 \mu_{12} \wp_{333}-4 \mu_{8} \wp_{133}-4 \mu_{6} \wp_{13} \wp_{333}+4 \mu_{6} \wp_{33} \wp_{133}-4 \wp_{12} \wp_{123} \\
& -2 \mu_{5} \wp_{13} \wp_{233}=0, \quad[-15]
\end{aligned}
$$

where the number in brackets [ ] indicates the weight. 
PROOF. We have already given the first two of these equations in the discussion following Corollary 5.5. Some of the others follow in the same way from the expansion of (5.2). Alternatively, some can be calculated directly by expressing the equations in Lemma 6.1 in terms of $\wp_{i j k \ell}$ and $\wp_{m n}$ functions, then using cross differentiation on suitably chosen pairs of equations. For example the first relation above for $\wp_{222}$ can be derived from

$$
\frac{\partial}{\partial u_{2}} \wp_{3333}-\frac{\partial}{\partial u_{3}} \wp_{2333}=0
$$

REMARK 6.6. For a fixed weight, these relations are not always unique, for example at weight -11 we also have the relation

$$
\begin{gathered}
\wp_{33} \wp_{122}+2 \wp_{23} \wp_{123}+3 \wp_{113}+\mu_{2} \wp_{13} \wp_{333}-\mu_{2} \wp_{33} \wp_{133} \\
+\mu_{8} \wp_{333}-2 \wp_{12} \wp_{233}-2 \mu_{4} \wp_{133}-\wp_{13} \wp_{223}=0 .
\end{gathered}
$$

These dual relations arise because in some cases the cross differentiation can be done in two different ways. In deriving the results in this section, it is sometimes required to make use of both bilinear relations at a given weight to provide enough equations to solve for the unknowns. A full list of the known bilinear relations is given at [15].

LEMMA 6.7. The quadratic expressions in the 3-index functions $\wp_{i j k}$ associated with (3.1) down to weight -23 can be expressed in terms of (at most cubic) relations in the $\wp_{m n}$ and $\wp_{1333}$. For example we have the following five relations down to weight -8 :

$$
\begin{aligned}
\wp_{333}{ }^{2}= & \wp_{33}{ }^{2} \mu_{1}{ }^{2}+2 \mu_{1} \wp_{23} \wp_{33}+\wp_{23}{ }^{2}+4 \wp_{13}-4 \wp_{33} \wp_{22}+4 \wp_{33}{ }^{3}-4 \mu_{2} \wp_{33}{ }^{2}-4 \mu_{4} \wp_{33}, \\
\wp_{233} \wp_{333}= & 2 \mu_{3} \wp_{33}{ }^{2}+4 \wp_{33}{ }^{2} \wp_{23}-\mu_{1} \wp_{33} \wp_{22}-2 \mu_{5} \wp_{33}-2 \mu_{2} \wp_{33} \wp_{23}+\mu_{1}{ }^{2} \wp_{33} \wp_{23}-2 \wp_{12} \\
& -\wp_{22} \wp_{23}+\mu_{1} \wp_{23}{ }^{2}+2 \mu_{1} \wp_{13}, \\
\wp_{133} \wp_{333}= & -\frac{1}{3} \mu_{1} \wp_{33} \wp_{12}+\frac{1}{3} \mu_{1}{ }^{2} \wp_{33} \wp_{13}-\frac{4}{3} \mu_{2} \wp_{33} \wp_{13}+\frac{2}{3} \wp_{33} \wp_{1333}-\frac{4}{3} \mu_{8} \wp_{33}+\wp_{23} \wp_{12} \\
& +\mu_{1} \wp_{13} \wp_{23}-2 \wp_{13} \wp_{22}, \\
\wp_{223} \wp_{333}= & 2 \mu_{1} \wp_{23} \wp_{22}-2 \mu_{2} \wp_{33} \wp_{22}+2 \mu_{1} \mu_{4} \wp_{23}-\mu_{1} \mu_{5} \wp_{33}+2 \wp_{33}{ }^{2} \wp_{22}-2 \mu_{4} \wp_{22}+2 \wp_{33} \wp_{23}^{2} \\
& +\frac{4}{3} \mu_{1}{ }^{2} \wp_{13}-\frac{4}{3} \mu_{2} \wp_{13}-\frac{4}{3} \mu_{1} \wp_{12}-\frac{4}{3} \mu_{8}-2 \wp_{22}{ }^{2}+\mu_{1} \mu_{2} \wp_{33} \wp_{23}+\frac{2}{3} \wp_{1333} \\
& +\wp_{23} \wp_{33} \mu_{3}+\mu_{1} \mu_{3} \wp_{33}{ }^{2}-\mu_{2} \wp_{23}{ }^{2}-\mu_{5} \wp_{23}, \\
\wp_{233}{ }^{2}= & 4 \wp_{33} \wp_{23}{ }^{2}+8 \wp_{13} \wp_{33}+4 \mu_{3} \wp_{33} \wp_{23}-2 \mu_{1} \wp_{23} \wp_{22}+\frac{4}{3} \mu_{1}{ }^{2} \wp_{13}-\frac{4}{3} \mu_{2} \wp_{13}+4 \mu_{6} \wp_{33} \\
& +\mu_{1}{ }^{2} \wp_{23}{ }^{2}-\frac{4}{3} \mu_{8}+\wp_{22}{ }^{2}-\frac{4}{3} \wp_{1333}-\frac{4}{3} \mu_{1} \wp_{12} .
\end{aligned}
$$


The expressions at lower weight quickly become very lengthy. For the purely trigonal case we give a list of the known quadratic expressions in the 3-index functions up to weight - 15 in Appendix B. The full list for the general $(3,4)$-curve down to weight -23 is available at [15].

PROOF. The relations can be found using a combination of three types of intermediate relations. One type is from terms in the expansion of (5.6). Another is to multiply one of the relations in Lemma 6.5 by another $\wp_{i j k}$ and substitute for previously calculated $\wp_{i j k} \wp_{\ell m n}$ relations of higher weight. Yet another is to take a derivative of one of the relations in Lemma 6.5 above and to substitute the known linear 4-index $\wp_{i j k \ell}$ and previously calculated $\wp_{i j k} \wp_{\ell m n}$ relations. Again, we work in a self-consistent way from higher to lower weights. The strategy for all the results in this section is to proceed down one weight at a time and to derive all the three types of relations (4-index $\wp_{i j k \ell}$, bilinear 2and 3-index, and quadratic 3-index) at a given weight before moving down to the next. An extra complication is that at certain weights some of the intermediate calculations can involve quartic terms in the $\wp_{m n}$ and $\wp_{1333}$. It is always possible to find enough relations to eliminate the quartic term up to weight -23 .

REMARK 6.8.

(1) These relations are the generalizations of the familiar relation $\left(\wp^{\prime}\right)^{2}=4 \wp^{3}-$ $g_{2} \wp-g_{3}$ in the genus 1 theory.

(2) For equations of weight below -23 , we have not been able to find cubic expressions for the $\wp_{i j k} \wp_{\ell m n}$ terms. We believe it should be possible to explain this using the results of Cho and Nakayashuiki [13], and we are currently investigating this possibility.

(3) The calculations in this section make no use of the expansion of the $\sigma$ function, which is given in the next section.

\section{Expansion of the $\sigma$-Function}

This section is devoted to show that the coefficient of the power series expansion of $\sigma(u)$ is a polynomial in $\mu_{j}$ s.

In the Weierstrass formulation of the theory of elliptic functions, the $\sigma$-function is defined as the power series expansion in the Abelian variable $u$ with coefficients depending on the Weierstrass parameters $g_{2}, g_{3}$, and related by certain recursive relations. 
The extension of Weierstrass theory to arbitrary algebraic curves was intensively developed in the 19th century and later, its development being attached to names such as Baker, Bolza, Brioschi, Burkhardt, Klein, and Wiltheiss. Some important modern developments of this theory are due to Buchstaber and Leykin $[7,8]$ who give a construction of linear differential (heat-like) operators that annihilate the $\sigma$-function for any $(m, n)$ curve. In the hyperelliptic case the operators are sufficient to find the recursion defining the whole series expansion. The exact analogue of the Weierstrass recursive series formula is known only for genus two, see [8], p. 68. In other cases the detailed results have not yet been developed, although the general method is provided in the publications mentioned above. Here we shall give the few first terms of the power series expansion, obtained by finding the coefficients of the Taylor series by using the PDEs given in Lemma 6.1.

THEOREM 7.1. The function $\sigma(u)$ associated with the general trigonal curve (2.1) of genus three has an expansion of the following form:

$$
\sigma\left(u_{1}, u_{2}, u_{3}\right)=\varepsilon \cdot\left(C_{5}\left(u_{1}, u_{2}, u_{3}\right)+C_{6}\left(u_{1}, u_{2}, u_{3}\right)+C_{7}\left(u_{1}, u_{2}, u_{3}\right)+\cdots\right),
$$

where $\varepsilon$ is a nonzero constant and each $C_{j}$ is a polynomial composed of sums of monomials in $u_{i}$ of odd total degree and of total weight $j$ with polynomial coefficient in $\mu_{i}$ s of total weight $(5-j)$. Especially, $\sigma(u)$ is an odd function (see 4.2). The first few $C_{j} s$ are

$$
\begin{aligned}
& C_{5}=u_{1}-u_{3} u_{2}{ }^{2}+\frac{1}{20} u_{3}{ }^{5}, C_{6}=\frac{1}{12} \mu_{1} u_{3}{ }^{4} u_{2}-\frac{1}{3} \mu_{1} u_{2}{ }^{3}, C_{7}=\frac{1}{504}\left(\mu_{1}{ }^{2}-3 \mu_{2}\right) u_{3}{ }^{7} \\
& +\frac{1}{6} \mu_{2} u_{3}{ }^{3} u_{2}{ }^{2}, \quad C_{8}=\frac{1}{360}\left(\mu_{1}{ }^{3}+9 \mu_{3}-2 \mu_{1} \mu_{2}\right) u_{3}{ }^{6} u_{2}-\frac{1}{2} \mu_{3} u_{3}{ }^{2} u_{2}{ }^{3}, \\
& C_{9}=\frac{1}{25920}\left(\mu_{1}{ }^{2}-3 \mu_{2}\right)^{2} u_{3}{ }^{9}+\frac{1}{120}\left(2 \mu_{4}-\mu_{2}{ }^{2}+\mu_{1}{ }^{2} \mu_{2}+6 \mu_{1} \mu_{3}\right) u_{3}{ }^{5} u_{2}{ }^{2} \\
& -\frac{1}{12}\left(4 \mu_{1} \mu_{3}+4 \mu_{4}+\mu_{2}^{2}\right) u_{3} u_{2}^{4}+\frac{1}{12} \mu_{4} u_{3}^{4} u_{1} \text {, } \\
& C_{10}=\frac{1}{20160}\left(8 \mu_{1} \mu_{4}-54 \mu_{2} \mu_{3}+3 \mu_{1} \mu_{2}{ }^{2}+18 \mu_{1}^{2} \mu_{3}+\mu_{1}{ }^{5}-12 \mu_{5}-4 \mu_{1}{ }^{3} \mu_{2}\right) u_{3}{ }^{8} u_{2} \\
& +\frac{1}{72}\left(6 \mu_{2} \mu_{3}+2 \mu_{1} \mu_{4}+\mu_{1} \mu_{2}^{2}+\mu_{1}^{2} \mu_{3}\right) u_{3}{ }^{4} u_{2}{ }^{3} \\
& -\frac{1}{60}\left(4 \mu_{1}^{2} \mu_{3}+\mu_{1} \mu_{2}^{2}+4 \mu_{5}+4 \mu_{1} \mu_{4}-2 \mu_{2} \mu_{3}\right) u_{2}{ }^{5}+\frac{1}{6} \mu_{5} u_{3}^{3} u_{2} u_{1} \text {, } \\
& C_{11}=-\frac{1}{6652800}\left(18 \mu_{1} \mu_{2} \mu_{3}+27 \mu_{1}{ }^{4} \mu_{2}-72 \mu_{6}-3 \mu_{1}{ }^{6}-24 \mu_{2} \mu_{4}+16 \mu_{1}{ }^{2} \mu_{4}-24 \mu_{1} \mu_{5}\right. \\
& \left.+27 \mu_{3}{ }^{2}+85 \mu_{2}{ }^{3}-4 \mu_{1}{ }^{3} \mu_{3}-82 \mu_{1}{ }^{2} \mu_{2}{ }^{2}\right) u_{3}{ }^{11}+\frac{1}{5040}\left(27 \mu_{3}{ }^{2}+\mu_{2}{ }^{3}-6 \mu_{2} \mu_{4}\right. \\
& \left.-18 \mu_{1} \mu_{2} \mu_{3}+8 \mu_{1}{ }^{3} \mu_{3}-4 \mu_{1} \mu_{5}+6 \mu_{1}{ }^{2} \mu_{4}+12 \mu_{6}+\mu_{1}{ }^{4} \mu_{2}-3 \mu_{1}{ }^{2} \mu_{2}{ }^{2}\right) u_{3}{ }^{7} u_{2}{ }^{2} \\
& -\frac{1}{72}\left(9 \mu_{3}^{2}-\mu_{2}^{3}-4 \mu_{2} \mu_{4}-2 \mu_{1} \mu_{2} \mu_{3}\right) u_{3}{ }^{3} u_{2}{ }^{4}
\end{aligned}
$$


J. C. Eilbeck et al.

$$
\begin{aligned}
& +\frac{1}{360}\left(\mu_{1} \mu_{5}-4 \mu_{2} \mu_{4}+\mu_{1}{ }^{2} \mu_{4}+3 \mu_{6}\right) u_{3}{ }^{6} u_{1}-\frac{1}{2} \mu_{6} u_{3}{ }^{2} u_{2}{ }^{2} u_{1}, \\
C_{12}= & -\frac{1}{1814400}\left(27 \mu_{1} \mu_{3}{ }^{2}-243 \mu_{2}{ }^{2} \mu_{3}-\mu_{1}{ }^{7}+72 \mu_{1} \mu_{2} \mu_{4}\right. \\
& -31 \mu_{1}{ }^{4} \mu_{3}-144 \mu_{2} \mu_{5}-16 \mu_{1}{ }^{3} \mu_{4} \\
& \left.+6 \mu_{1}{ }^{5} \mu_{2}-10 \mu_{1}{ }^{3} \mu_{2}{ }^{2}+24 \mu_{1}{ }^{2} \mu_{5}+4 \mu_{1} \mu_{2}{ }^{3}-72 \mu_{1} \mu_{6}+180 \mu_{1}{ }^{2} \mu_{2} \mu_{3}\right) u_{3}{ }^{10} u_{2} \\
& +\frac{1}{2160}\left(18 \mu_{3} \mu_{4}-2 \mu_{1} \mu_{2}{ }^{3}+27 \mu_{1} \mu_{3}{ }^{2}-9 \mu_{2}{ }^{2} \mu_{3}+\mu_{1}{ }^{3} \mu_{2}{ }^{2}+\mu_{1}{ }^{4} \mu_{3}+6 \mu_{1}{ }^{2} \mu_{2} \mu_{3}\right. \\
& \left.+2 \mu_{1}{ }^{3} \mu_{4}+12 \mu_{1} \mu_{6}\right) u_{3}{ }^{6} u_{2}{ }^{3}-\frac{1}{24} \mu_{3}\left(3 \mu_{1} \mu_{3}+4 \mu_{4}+\mu_{2}{ }^{2}\right) u_{3}{ }^{2} u_{2}{ }^{5} \\
& +\frac{1}{120}\left(6 \mu_{3} \mu_{4}+2 \mu_{1} \mu_{6}-\mu_{2} \mu_{5}+\mu_{1}{ }^{2} \mu_{5}\right) u_{3}{ }^{5} u_{2} u_{1} \\
& -\frac{1}{6}\left(2 \mu_{1} \mu_{6}+2 \mu_{3} \mu_{4}+\mu_{2} \mu_{5}\right) u_{3} u_{2}{ }^{3} u_{1} .
\end{aligned}
$$

PROOF. We divide the proof into four parts.

Step 1. We have already shown in Lemma 4.2, that all the terms are of total odd degree or even degree. We first show that the expansion contains a term linear in $u_{1}$, so the expansion must be odd.

Let $B(D)$ be the Brill-Noether matrix for an effective divisor $D$ of $C$. Then it is well known that (see for example [24] or [26])

$$
\operatorname{dim} \Gamma(C, \mathcal{O}(D))=\operatorname{deg} D+1-\operatorname{rank} B(D)
$$

where $\Gamma(C, \mathcal{O}(D))$ is the space of functions on $C$ whose divisors are larger than or equal to $-D$. Moreover, for two points $P_{1}, P_{2}$ on $C, \operatorname{dim} \Gamma\left(C, \mathcal{O}\left(P_{1}+P_{2}\right)\right)>1$ if and only if the point $\iota\left(P_{1}, P_{2}\right) \in \Theta^{[2]}$ is a nonsingular point of $\Theta^{[2]}$ (note that $C$ is of genus 3 ). By checking the Brill-Noether matrix $B\left(P_{1}+P_{2}\right)$, we see $\Theta^{[2]}$ is nonsingular everywhere. Especially $\kappa^{-1}\left(\Theta^{[2]}\right)$ is nonsingular at the origin $(0,0,0)$. On the other hand, let $u$ and $v$ be two variables on $\kappa^{-1}\left(\Theta^{[1]}\right)$. Then we have an expansion with respect to $v_{3}$ :

$$
0=\sigma(u+v)=\sigma_{3}(u) v_{3}+\frac{1}{2}\left(\sigma_{2}(u)+\sigma_{33}(u)\right) v_{3}^{2}+\cdots,
$$

where $\sigma_{i}=\partial \sigma / \partial u_{i}$, etc. Hence

$$
\sigma_{3}(u)=0 \quad \sigma_{2}(u)+\sigma_{33}(u)=0 .
$$

Again by expansion

$$
0=\sigma_{3}(u)=\sigma_{33}(0) v_{3}+\cdots,
$$


we see that

$$
\sigma_{33}(0)=0
$$

In summary,

$$
\sigma_{3}(0)=\sigma_{2}(0)=0
$$

So from the above arguments and (4.14), we must have

$$
\sigma_{1}(0) \neq 0
$$

Hence the $\sigma$-expansion must be odd.

Step 2. Next we show that the terms of weight less than 5 vanish and $C_{5}(u)$ is nontrivial. We write all the possible odd terms up to and including terms of weight 5 . Using the first two equations in 6.1, we can show that the coefficients of the terms of weight four and less are zero, and that the coefficients of weight 5 are given by those in $C_{5}$ up to multiplication by a constant. We know from Step 1 that this constant is nonzero and we insert this constant into the $\varepsilon$.

Step 3. We now calculate the coefficients $C_{i}, i>5$. The proof of this step is by construction (with heavy use of Maple) using the PDEs given in Lemma 6.1. We expand $\sigma\left(u_{1}, u_{2}, u_{3}\right)$ in a Taylor series with undetermined coefficients, keeping only odd terms. We do not assume that the coefficients of the expansion are polynomial in the $\mu_{i}$, only that they are independent of the $u_{i}$. We then insert the expansion into the 4-index PDEs for the $\wp$, and truncate to successive orders in the weights of the $u_{i}$. These give a series of linear equations for the coefficients, and by using a sufficient number of the PDEs we can always find unique solutions, as listed above. We have carried out this calculation down to $C_{18}$. We have omitted the details of the expressions for $C_{13}, \ldots, C_{18}$, as they are rather lengthy, but these are available at [15].

Step 4. Now consider the general term in the expansion. Set

$$
A u_{1}{ }^{p} u_{2}^{q} u_{3}^{r}, \quad A \in \mathbf{Q}\left(\mu_{i}\right)
$$

to be the lowest weight unknown term. Since we have already shown by construction that the coefficients for all weights down to -29 with respect to $u_{j}$ s are polynomials, we may assume that $p+q+r \geqq 4$. Let $(\sharp)$ be the set of quadratic equations in $\sigma(u)$ and its (higher) 
derivatives obtained from Lemma 6.1 by multiplying by $\sigma(u)^{2}$. We take an equation

$$
\sigma(u)^{2} Q_{i j k \ell}(u)=\cdots
$$

from (\#) such that $u_{1}{ }^{p} u_{2}{ }^{q} u_{3}{ }^{r}$ is divisible by $u_{i} u_{j} u_{k} u_{\ell}$. We have at least one such equation. Differentiating (7.2), we have an equation of the form

$$
\sigma(u)\left(\frac{\partial^{p+q+r} \sigma}{\partial u_{1}^{p} \partial u_{1}^{q} \partial u_{1}^{r}}\right)(u)+\cdots=0
$$

such that all terms are polynomial of $\sigma(u)$ and its higher derivatives and such that

$$
\left(\frac{\partial^{p+q+r} \sigma}{\partial u_{1}^{p} \partial u_{1}^{q} \partial u_{1}^{r}}\right)(u)
$$

is the highest derivative in (7.3). By looking at the coefficient of the term $u_{1}$, we have a linear equation of the form

$$
A+\cdots=0
$$

over $\mathbf{Q}\left[\mu_{1}, \cdots, \mu_{12}\right]$. Since the other terms except $A$ in the above equation come from terms of $\sigma(u)$ whose weight is less than weight of $u_{1}{ }^{p} u_{2}{ }^{q} u_{3}{ }^{r}$, we see $A$ is a polynomial in the $\mu_{j}$ s by the induction hypothesis.

\section{REMARK 7.2.}

(1) In Theorem 7.1, the constant $\varepsilon$ might be unity, another 8th root of 1 , or some other constant. We have not been able to narrow down this result. If the case $\varepsilon=1$ is true, then the determination of $\varepsilon$ reduces to the choice of roots in (2.14) and (4.9). The remaining results in this article do not depend on this choice, or on the possibility that $\varepsilon \neq 1$.

(2) The weight of $\sigma(u)$ is inferred from (4.9) since the weight of $\left|\omega^{\prime}\right|$ is $5+2+1$ and the conjectured weight of $D$ is 72 . The weight of the terms in the exponentials are all 0 and the weight of $c$ is $72 / 8-(5+2+1) / 2=5$ and coincides with the terms in the expansion of 7.1 if the weight of $\varepsilon$ is 0 .

We shall need the following special property of the $\sigma$-function in the purely trigonal case later on: 
LEMMA 7.3. The $\sigma$ function associated with the purely trigonal curve (3.1) satisfies $\sigma([-\zeta] u)=-\zeta \sigma(u)$ for $u \in \mathbb{C}^{3}$ under the notation (3.1).

PROOF. Since $\Lambda$ is stable under the action of $[\zeta]$ and $[-1]$, we can check the statement by Lemma 4.1 and Remark 4.3.

\section{Basis of the Space $\Gamma\left(J, \mathcal{O}\left(n \Theta^{[2]}\right)\right)$}

For notational simplicity, we denote

$$
\partial_{j}=\frac{\partial}{\partial u_{j}} .
$$

We also define

$$
\wp^{[i j]}=\text { the determinant of the }(i, j) \text {-(complementary) minor of }\left[\wp_{i j}\right]_{3 \times 3} \text {. }
$$

We have explicit bases of the vector spaces $\Gamma\left(J, \mathcal{O}\left(2 \Theta^{[2]}\right)\right)$ and $\Gamma\left(J, \mathcal{O}\left(3 \Theta^{[2]}\right)\right)$ as follows (see also [13], Example in Section 9):

LEMMA 8.1. We have the following:

$$
\begin{aligned}
\Gamma\left(J, \mathcal{O}\left(2 \Theta^{[2]}\right)\right)= & \mathbb{C} 1 \oplus \mathbb{C} \wp_{11} \oplus \mathbb{C} \wp_{12} \oplus \mathbb{C} \wp_{13} \oplus \mathbb{C} \wp_{22} \oplus \mathbb{C} \wp_{23} \oplus \mathbb{C} \wp_{33} \oplus \mathbb{C} Q_{1333}, \\
\Gamma\left(J, \mathcal{O}\left(3 \Theta^{[2]}\right)\right)= & \Gamma\left(J, \mathcal{O}\left(2 \Theta^{[2]}\right)\right) \oplus \mathbb{C} \wp_{111} \oplus \mathbb{C} \wp_{112} \oplus \mathbb{C} \wp_{113} \oplus \mathbb{C} \wp_{122} \oplus \mathbb{C} \wp_{123} \\
& \oplus \mathbb{C} \wp_{133} \oplus \mathbb{C} \wp_{222} \oplus \mathbb{C} \wp_{223} \oplus \mathbb{C} \wp_{233} \oplus \mathbb{C} \wp_{333} \oplus \mathbb{C} \partial_{1} Q_{1333} \\
& \oplus \mathbb{C} \partial_{2} Q_{1333} \oplus \mathbb{C} \partial_{3} Q_{1333} \\
& \oplus \mathbb{C} \wp^{[11]} \oplus \mathbb{C} \wp^{[12]} \oplus \mathbb{C} \wp^{[13]} \oplus \mathbb{C} \wp^{[22]} \oplus \mathbb{C} \wp^{[23]} \oplus \mathbb{C} \wp^{[33]} .
\end{aligned}
$$

PROOF. We know the dimensions of the spaces above are $2^{3}=8$ and $3^{3}=27$, respectively by the Riemann-Roch theorem for Abelian varieties (see for example, [23], (pp. 150155), [20], (p. 99, Theorem 4.1)). Moreover, (4.14) shows that the functions in the right hand sides belong to the spaces of the left hand sides, respectively. For the space $\Gamma\left(J, \mathcal{O}\left(2 \Theta^{[2]}\right)\right), \wp_{i j}$ and $Q_{i j k \ell}$ become the basis of the space from Definition 5.1, Lemma 4.1, and the arguments in the previous section. However these are not all linearly independent, since there are connecting relations, such as those given in Lemma 6.1, and the number of these relations is greater than the dimension of the space. Thus the 
problem is reduced to picking the linearly independent bases as a function space. It is obvious that such independence does not depend upon the coefficients of curve by considering these expansions around the origin of $\mathbb{C}^{3}$. Hence by multiplying by $\sigma(u)^{2}$ from the right hand side with respect to $u_{1}, u_{2}, u_{3}$, and after putting all the $\mu_{j}$ equal to zero, we see the functions of the right hand side are linearly independent. The authors used a computer to check this. Similarly, for the space $\Gamma\left(J, \mathcal{O}\left(3 \Theta^{[2]}\right)\right)$, the 27 functions obtained by multiplying by $\sigma(u)^{3}$ from the right hand side are checked to be linearly independent by using a computer, expanding the given functions in the Abelian variables (cf. Theorem 7.1) to a sufficiently high power that independence is checked. We also see both decompositions in Lemma 8.1 in Example in Section 9 of [13].

\section{The First Main Addition Theorem}

THEOREM 9.1. The $\sigma$-function associated with (2.1) satisfies the following addition formula on $J \times J$ :

$$
\begin{aligned}
- & \frac{\sigma(u+v) \sigma(u-v)}{\sigma(u)^{2} \sigma(v)^{2}}=\wp_{11}(u)-\wp_{11}(v)+\wp_{12}(u) \wp_{23}(v)-\wp_{12}(v) \wp_{23}(u) \\
& +\wp_{13}(u) \wp_{22}(v)-\wp_{13}(v) \wp_{22}(u)+\frac{1}{3}\left(\wp_{33}(u) Q_{1333}(v)-\wp_{33}(v) Q_{1333}(u)\right) \\
& -\frac{1}{3} \mu_{1}\left(\wp_{12}(u) \wp_{33}(v)-\wp_{12}(v) \wp_{33}(u)\right)-\mu_{1}\left(\wp_{13}(u) \wp_{23}(v)-\wp_{13}(v) \wp_{23}(u)\right) \\
& +\frac{1}{3}\left(\mu_{1}{ }^{2}-\mu_{2}\right)\left(\wp_{13}(u) \wp_{33}(v)-\wp_{13}(v) \wp_{33}(u)\right)+\frac{1}{3} \mu_{8}\left(\wp_{33}(u)-\wp_{33}(v)\right) .
\end{aligned}
$$

PROOF. Firstly, we notice that the left hand side is an odd function with respect to $u \leftrightarrow v$, and that it has poles of order 2 along $\left(\Theta^{[2]} \times J\right) \cup\left(J \times \Theta^{[2]}\right)$ but nowhere else. Moreover it is of weight -10 . Therefore, by Lemma 8.1, the left hand side is expressed by a finite sum of the form

$$
\sum_{j} A_{j}\left(X_{j}(u) Y_{j}(v)-X_{j}(v) Y_{j}(u)\right)
$$

where the $A_{j}$ are rational functions of the $\mu_{i}$ s with homogeneous weight, and the $X_{j}$ and $Y_{j}$ are functions chosen from the right hand side of the first equality in Lemma 8.1. We claim that all the $A_{j}$ are polynomial in the $\mu_{i}$ s. Suppose all the $A_{j}$ s are reduced fractional expressions, and at least one of the $A_{j} \mathrm{~s}$ is not a polynomial. Take the least common multiple $B$ of all the denominators of the $A_{j}$ s. Note that there is a set of special values of the $\mu_{i}$ s such that $B$ vanishes and the numerator of at least one $A_{j}$ does not vanish. After 
multiplying the equation "lhs" $=(9.2)$ by $B \sigma(u)^{2} \sigma(v)^{2}$, and taking the $\mu_{i}$ s to be such a zero of $B$, we have a contradiction, by using the linear independence of Lemma 8.1 twice with respect to the variables $u$ and $v$ for the corresponding curve of (2.1). Hence, all the $A_{j}$ must be polynomials. Hence, we see that the desired right hand side must be expressed by using constants $a, b, c, d, e, f, g_{1}, g_{2}, h_{1}, h_{2}, i_{1}, i_{2}, j, k_{1}, k_{2}, k_{3}$ which are polynomials in $\mu_{i}$ s and independent of the $u_{i}$ and $v_{i}$, as follows:

$$
\begin{aligned}
a & {\left[\wp_{11}(u)-\wp_{11}(v)\right]+b\left[\wp_{12}(u) \wp_{23}(v)-\wp_{12}(v) \wp_{23}(u)\right]+c\left[\wp_{13}(u) \wp_{22}(v)-\wp_{13}(v) \wp_{22}(u)\right] } \\
& +d\left[Q_{1333}(u) \wp_{33}(v)-Q_{1333}(v) \wp_{33}(u)\right]+e\left[\wp_{12}(u) \wp_{33}(v)-\wp_{12}(v) \wp_{33}(u)\right] \\
& +f\left[\wp_{13}(u) \wp_{23}(v)-\wp_{13}(v) \wp_{23}(u)\right]+g_{1}\left[\wp_{13}(u) \wp_{33}(v)-\wp_{13}(v) \wp_{33}(u)\right] \\
& +g_{2}\left[Q_{1333}(u)-Q_{1333}(v)\right]+h_{1}\left[\wp_{23}(u) \wp_{22}(v)-\wp_{23}(v) \wp_{22}(u)\right]+h_{2}\left[\wp_{12}(u)-\wp_{12}(v)\right] \\
& +i_{1}\left[\wp_{22}(u) \wp_{33}(v)-\wp_{22}(v) \wp_{33}(u)\right]+i_{2}\left[\wp_{13}(u)-\wp_{13}(v)\right]+j\left[\wp_{23}(u) \wp_{33}(v)\right. \\
& \left.-\wp_{23}(v) \wp_{33}(u)\right]+k_{1}\left[\wp_{22}(u)-\wp_{22}(v)\right]+k_{2}\left[\wp_{23}(u)-\wp_{23}(v)\right]+k_{3}\left[\wp_{33}(u)-\wp_{33}(v)\right] .
\end{aligned}
$$

We find by computer using Maple, on substituting the expansion (7.1) up to $C_{13}$ terms of $\sigma(u)$ into (9.3), and truncating up to weight 18 in the $u_{i}$ and $v_{i}$, that

$$
\begin{aligned}
& a=b=c=-1, \quad d=\frac{1}{3}, \quad e=-\frac{1}{3} \mu_{1}, \quad f=-\mu_{1}, \quad g_{1}=\frac{1}{3}\left(\mu_{1}^{2}-\mu_{2}\right), \\
& g_{2}=h_{1}=h_{2}=i_{1}=i_{2}=j=k_{1}=k_{2}=0, \quad k_{3}=\frac{1}{3} \mu_{8},
\end{aligned}
$$

as asserted. In the Maple calculation, it is not necessary to assume the polynomial nature of the coefficients as functions of the $\mu_{j}$.

REMARK 9.2. By applying

$$
\frac{1}{2} \frac{\partial}{\partial u_{i}}\left(\frac{\partial}{\partial u_{j}}+\frac{\partial}{\partial v_{j}}\right) \log
$$

to 9.1, we have $-\wp_{i j}(u+v)+\wp_{i j}(u)$ from the left hand side, and have a rational expression of several $\wp_{i j \ldots \ell}(u) s$ and $\wp_{i j \ldots \ell}(v) s$ on the right hand side. Hence, we have an algebraic addition formula for $\wp_{i j}(u)$. 
REMARK 9.3. By putting $v=u-(\delta, 0,0)$ and letting $\delta \rightarrow 0$, we can get a "double-angle" $\sigma$-formula

$$
\begin{aligned}
& \frac{\sigma(2 u)}{\sigma(u)^{4}}=-\wp_{111}(u)-\wp_{112} \wp_{23}+\wp_{12}(u) \wp_{123}(u)-\wp_{113}(u) \wp_{22}(u)+\wp_{13}(u) \wp_{122}(u) \\
& \quad-\frac{1}{3} \wp_{133}(u) Q_{1333}(u)+\frac{1}{3} \wp_{33}(u) \frac{\partial}{\partial u_{1}} Q_{1333}(u)+\frac{1}{3} \mu_{1}\left(\wp_{112}(u) \wp_{33}(u)-\wp_{12}(u) \wp_{133}(u)\right) \\
& \quad+\mu_{1}\left(\wp_{113}(u) \wp_{23}-\wp_{13}(u) \wp_{123}\right)-\frac{1}{3}\left(\mu_{1}^{2}-\mu_{2}\right)\left(\wp_{113}(u) \wp_{33}(u)\right. \\
& \left.\quad-\wp_{13}(u) \wp_{133}(u)\right)-\frac{1}{3} \mu_{8} \wp_{133}(u) .
\end{aligned}
$$

In the case of the elliptic curve, the corresponding relation is $\sigma(2 u)=-\wp^{\prime}(u) \sigma^{4}(u)$, whilst the corresponding formula for the hyperelliptic genus two curve is given in [4], p. 129.

\section{The Second Main Addition Theorem}

The second main addition result applies only in the purely trigonal case (3.1), using the results of Lemma 7.3. The formula is as follows:

THEOREM 10.1. The $\sigma$-function associated with (3.1) satisfies the following addition formula on $J \times J$ :

$$
\frac{\sigma(u+v) \sigma(u+[\zeta] v) \sigma\left(u+\left[\zeta^{2}\right] v\right)}{\sigma(u)^{3} \sigma(v)^{3}}=R(u, v)+R(v, u)
$$

where

$$
\begin{aligned}
& R(u, v)=-\frac{1}{3} \wp_{13}(u) \partial_{3} Q_{1333}(v)-\frac{3}{4} \wp_{23}(u) \wp_{112}(v)-\frac{1}{2} \wp_{111}(u)+\frac{1}{4} \wp_{122}(u) \wp^{[11]}(v) \\
& \quad-\frac{1}{4} \wp_{222}(u) \wp^{[12]}(v)+\frac{1}{12} \partial_{3} Q_{1333}(u) \wp^{[11]}(v)+\frac{1}{2} \wp_{333}(u) \wp^{[22]}(v)-\frac{1}{4} \mu_{3} \wp_{333}(u) \wp^{[12]}(v) \\
& \quad+\frac{1}{2} \mu_{6} \wp_{13}(u) \wp_{333}(v)-\frac{1}{4} \mu_{9} \wp_{23}(u) \wp_{333}(v)-\frac{1}{2} \mu_{12} \wp_{333}(u) .
\end{aligned}
$$

PROOF. Our goal is to express

$$
\frac{\sigma(u+v) \sigma(u+[\zeta] v) \sigma\left(u+\left[\zeta^{2}\right] v\right)}{\sigma(u)^{3} \sigma(v)^{3}}
$$

using several $\wp$ functions. Because $(10.2)$ belongs to $\Gamma\left(J \times J, \mathcal{O}\left(3\left(\left(\Theta^{[2]} \times J\right) \cup\left(J \times \Theta^{[2]}\right)\right)\right)\right)$, a similar argument to that at the beginning of the proof of Theorem 9.1 shows that it must 
be a finite sum of multi-linear forms of the 27 functions in Lemma 8.1, namely, of the form

$$
\sum_{j}^{\text {finite sum }} C_{j} X_{j}(u) Y_{j}(v) \text {, }
$$

where $X_{j}$ and $Y_{j}$ are any of the functions appearing in the right hand side of the description of $\Gamma\left(J, \mathcal{O}\left(3 \Theta^{[2]}\right)\right)$ in Lemma 8.1, and the $C_{j}$ are polynomial in $\mu_{i}$ s. Moreover, (10.2) has the following properties:

L1. As a function on $J \times J$, its weight is $(-5) \times 3=-15$;

L2. It is invariant under $u \mapsto[\zeta] u$ (resp. $v \mapsto[\zeta] v)$;

L3. It has a pole of order 3 on $\left(\Theta^{[2]} \times J\right) \cup\left(J \times \Theta^{[2]}\right)$;

L4. It is invariant under the exchange $u \leftrightarrow v$ (by Lemma 7.3).

Hence, (10.3) has the same properties. Thus, we may consider only the functions in our basis of $\Gamma\left(J, \mathcal{O}\left(3 \Theta^{[2]}\right)\right)$ that have the following corresponding properties:

$\mathrm{R} 1$. The weight is greater than or equal to $(-5) \times 3=-15$;

R2. They are invariant under $u \mapsto[\zeta] u$;

R3. They have poles of order at most 3 on $\Theta^{[2]}$.

There are 12 such functions and they are listed as follows:

$$
\begin{array}{llll}
1, & \wp_{13} \quad(\text { weight }=-6), & \wp_{23} \quad(\text { weight }=-3), \\
\wp_{111} \quad(\text { weight }=-15), & \wp_{112} \quad(\text { weight }=-12), & \wp_{122} \quad(\text { weight }=-9), \\
\wp_{222} \quad(\text { weight }=-6), & \wp_{333} \quad(\text { weight }=-3), & \wp^{[22]} \quad(\text { weight }=-12), \\
\wp^{[12]} \quad(\text { weight }=-9), & \wp^{[11]} \quad(\text { weight }=-6), & \\
\partial_{3} Q_{1333}=-6\left(\wp_{13} \wp_{333}-\wp_{133} \wp_{33}\right)-3 \wp_{122} \quad(\text { weight }=-9),
\end{array}
$$

and the $\wp^{[i j]}$ are defined in (8.2). Here the last equality is given by cross-differentiation from $\partial_{1} Q_{3333}$ using the first of the relations in Lemma 6.1 with $\mu_{1}=\mu_{2}=\mu_{4}=0$. Since (10.2) is an even function, it must be of the form

$$
\frac{\sigma(u+v) \sigma(u+[\zeta] v) \sigma\left(u+\left[\zeta^{2}\right] v\right)}{\sigma(u)^{3} \sigma(v)^{3}}=\tilde{R}(u, v)+\tilde{R}(v, u)
$$


where

$$
\begin{aligned}
& \tilde{R}(u, v)=a_{1} \wp_{13}(u) \wp_{122}(v)+a_{2} \wp_{13}(u) \partial_{3} Q_{1333}(v)+a_{3} \wp_{23}(u) \wp_{112}(v)+a_{4} \wp_{111}(u) \\
& \quad+a_{5} \wp_{122}(u) \wp^{[11]}(v)+a_{6} \wp_{222}(u) \wp^{[12]}(v)+a_{7} \partial_{3} Q_{1333}(u) \wp^{[11]}(v)+a_{8} \wp_{333}(u) \wp^{[22]}(v) \\
& +b_{1} \wp_{13}(u) \wp_{222}(v)+b_{2} \wp_{23}(u) \wp_{122}(v)+b_{3} \wp_{23}(u) \partial_{3} Q_{1333}(v) \\
& \quad+b_{4} \wp_{112}(u)+b_{5} \wp_{222}(u) \wp^{[11]}(v)+b_{6} \wp_{333}(u) \wp^{[12]}(v) \\
& \quad+c_{1} \wp_{13}(u) \wp_{333}(v)+c_{2} \wp_{23}(u) \wp_{222}(v)+c_{3} \wp_{122}(u)+c_{4} \wp_{333}(u) \wp^{[11]}(v) \\
& \quad+c_{5} \partial_{3} Q_{1333}(u)+d_{1} \wp_{23}(u) \wp_{333}(v)+d_{2} \wp_{222}(u)+e_{1} \wp_{333}(u) .
\end{aligned}
$$

By substituting (7.1) into (10.4), and comparing coefficients of different mononomials in $u_{i}, v_{j}$, we can find the constants $a_{1}, \cdots, e_{1}$ depending on the $\mu_{k} \mathrm{~s}$. Again, in this lengthy Maple calculation, it is not necessary to assume the coefficients are polynomial in the $\mu_{i}$.

REMARK 10.2. By applying

$$
\frac{1}{3}\left(\frac{\partial^{2}}{\partial u_{i} \partial u_{j}}+\frac{\partial^{2}}{\partial u_{i} \partial v_{j}}+\frac{\partial^{2}}{\partial v_{i} \partial v_{j}}\right) \log
$$

to (10.1), we obtain algebraic addition formulae for standard Abelian functions, which would be interesting to compare with those of Remark 9.2.

REMARK 10.3. By putting $v=-u+(\delta, 0,0)$ into (10.1), dividing through by $\delta$ and letting $\delta \rightarrow 0$, we can get an unusual "shifted" $\sigma$-formula of the form

$$
-\frac{\sigma(u-[\zeta] u) \sigma\left(u-\left[\zeta^{2}\right] u\right)}{\sigma(u)^{6}}=\sum_{i=1}^{12} c_{i}\left[g_{i}(u) \partial_{1} f_{i}(u)-f_{i}(u) \partial_{1} g_{i}(u)\right]
$$

where the $f_{i}$ and the $g_{i}$ are the even and odd derivative components respectively of the formula in (10.1), i.e. as given in the following table

\begin{tabular}{c|c|c||c|c|c}
$c_{i}$ & $f_{i}$ & $g_{i}$ & $c_{i}$ & $f_{i}$ & $g_{i}$ \\
\hline$\frac{1}{2}$ & $\wp_{13}(u)$ & $\wp_{122}(u)$ & $-\frac{1}{3}$ & $\wp_{13}(u)$ & $\partial_{3} Q_{1333}(u)$ \\
$-\frac{3}{4}$ & $\wp_{23}(u)$ & $\wp_{112}(u)$ & $-\frac{1}{2}$ & 1 & $\wp_{111}(u)$ \\
$\frac{1}{4}$ & $\wp^{[11]}(u)$ & $\wp_{122}(u)$ & $-\frac{1}{4}$ & $\wp^{[12]}(u)$ & $\wp_{222}(u)$ \\
$\frac{1}{12}$ & $\wp^{[11]}(v)$ & $\partial_{3} Q_{1333}(u)$ & $\frac{1}{2}$ & $\wp^{[22]}(u)$ & $\wp_{333}(u)$ \\
$-\frac{1}{4} \mu_{3}$ & $\wp^{[12]}(u)$ & $\wp_{333}(u)$ & $\frac{1}{2} \mu_{6}$ & $\wp_{13}(u)$ & $\wp_{333}(u)$ \\
$-\frac{1}{4} \mu_{9}$ & $\wp_{23}(u)$ & $\wp_{333}(v)$ & $-\frac{1}{2} \mu_{12}$ & 1 & $\wp_{333}(u)$
\end{tabular}


REMARK 10.4. In the general elliptic case, there appears to be no formulae corresponding to (10.1) and (10.6). However for the specialized equianharmonic case, where $\wp$ satisfies

$$
\left(\wp^{\prime}\right)^{2}=4 \wp^{3}-g_{3}
$$

it is straightforward to show that

$$
\frac{\sigma(u+v) \sigma(u+\zeta v) \sigma\left(u+\zeta^{2} v\right)}{\sigma^{3}(u) \sigma^{3}(v)}=-\frac{1}{2}\left(\wp^{\prime}(u)+\wp^{\prime}(v)\right)
$$

and

$$
\frac{\sigma((1-\zeta) u) \sigma\left(\left(1-\zeta^{2}\right) u\right)}{\sigma^{6}(u)}=3 \wp^{2}(u)
$$

These seem to be just the first of a family of multi-term addition formulae on special curves with automorphisms, which will be discussed in more detail elsewhere.

\section{Appendix}

\section{A The Fundamental Bi-differential}

We write the polynomial $f(x, y)$ in (2.1) that defines the trigonal curve $C$ as

$$
f(x, y)=y^{3}+p(x) y^{2}+q(x) y-r(x)
$$

with

$$
p(x)=\mu_{1} x+\mu_{4}, \quad q(x)=\mu_{2} x^{2}+\mu_{5} x+\mu_{8}, \quad r(x)=x^{4}+\mu_{3} x^{3}+\mu_{6} x^{2}+\mu_{9} x+\mu_{12} .
$$

We describe explicitly the fundamental nonnormalized bi-differential (Klein's fundamental 2 -form of the second kind) $\Sigma((x, y),(z, w))$ in (2.18) of the curve for $(x, y),(z, w)$ in $C$ defined by $f(x, y)=0$.

Following the scheme described in [1] and applied to trigonal curves in [16], [10] and the present article, one can realize $\Sigma((x, y),(z, w))$ explicitly as

$$
\Omega((x, y),(z, w))=\frac{F((x, y),(z, w)) d x d z}{(x-z)^{2} f_{Y}(x, y) f_{W}(z, w)}
$$


with the polynomial $F((x, y) ;(z, w))$ given by the formula

$$
\begin{aligned}
& F((x, y),(z, w))=(w y+Q(x, z))(w y+Q(z, x)) \\
& \quad+w\left(w\left[\frac{f(x, y)}{y}\right]_{y}+T(x, z)\right)+y\left(y\left[\frac{f(z, w)}{w}\right]_{W}+T(z, x)\right)-F_{0}(x, z)
\end{aligned}
$$

with

$$
\begin{aligned}
& Q(x, z)=\left(\mu_{1}^{2}-\mu_{2}\right) x z+\left(2 \mu_{1} \mu_{4}-\mu_{5}\right) x-\mu_{8}+\mu_{4}^{2} \\
& T(x, z)=3 \mu_{12}+(z+2 x) \mu_{9}+x(x+2 z) \mu_{6}+3 \mu_{3} x^{2} z+p(z) q(x)+x^{2} z^{2}+2 x^{3} z .
\end{aligned}
$$

The term $F_{0}(x, y)$ vanishes at $\mu_{1}=\mu_{4}=0$ and is given by

$$
\begin{aligned}
F_{0}(x, y) & =c_{32}(x+z) x^{2} z^{2}+c_{22} x^{2} z^{2}+c_{21}(x+z) x z+c_{11} x z+c_{10}(x+z)+c_{00}, \\
c_{32} & =-\mu_{1}, \quad c_{22}=-2 \mu_{4}-2 \mu_{1}^{2} \mu_{2}+\mu_{1}^{4}+2 \mu_{3} \mu_{1}, \\
c_{21} & =\mu_{6} \mu_{1}-2 \mu_{1} \mu_{4} \mu_{2}+\mu_{3} \mu_{4}-\mu_{5} \mu_{1}^{2}+2 \mu_{1}^{3} \mu_{4}, \\
c_{11} & =2\left(3 \mu_{1}{ }^{2} \mu_{4}{ }^{2}+\mu_{6} \mu_{4}+\mu_{9} \mu_{1}-2 \mu_{1} \mu_{4} \mu_{5}-\mu_{1}{ }^{2} \mu_{8}-\mu_{2} \mu_{4}^{2}\right) \\
C_{10} & =-\mu_{5} \mu_{4}{ }^{2}+\mu_{1} \mu_{12}+2 \mu_{1} \mu_{4}^{3}-2 \mu_{1} \mu_{4} \mu_{8}+\mu_{3} \mu_{4}, \\
c_{00} & =\mu_{4}\left(\mu_{4}{ }^{3}+2 \mu_{12}-2 \mu_{4} \mu_{8}\right) .
\end{aligned}
$$

We also remark that the Expression (A.3) generalizes the Kleinian 2-polar previously derived in the hyperelliptic case [1].

\section{B Quadratic 3-Index Relations}

A complete list of the known relations quadratic in 3-index $\wp_{i j k}$, up to weight -15 , for the "purely trigonal" case is given below. Note that with care we can obtain an expression such that the highest power on the r.h.s. is no more than cubic. The number in square brackets [ ] is the weight. A fuller list for the general $(3,4)$ case is given at $[15]$.

$$
\begin{aligned}
\wp_{333}{ }^{2} & =\wp_{23}^{2}+4 \wp_{13}-4 \wp_{33} \wp_{22}+4 \wp_{33}{ }^{3}, \quad[-6] \\
\wp_{233} \wp_{333} & =-\wp_{22} \wp_{23}+4 \wp_{23} \wp_{33}{ }^{2}+2 \mu_{3} \wp_{33}{ }^{2}-2 \wp_{12}, \quad[-7] \\
\wp_{233}{ }^{2} & =4 \wp_{33} \wp_{23}{ }^{2}+4 \mu_{3} \wp_{33} \wp_{23}+\wp_{22}{ }^{2}-\frac{4}{3} \wp_{1333}+4 \mu_{6} \wp_{33}+8 \wp_{33} \wp_{13}, \quad[-8] \\
\wp_{333} \wp_{223} & =2 \wp_{33} \wp_{23}{ }^{2}+\mu_{3} \wp_{33} \wp_{23}-2 \wp_{22}{ }^{2}+\frac{2}{3} \wp_{1333}+2 \wp_{33}{ }^{2} \wp_{22}, \quad[-8]
\end{aligned}
$$




$$
\begin{aligned}
& \wp_{223} \wp_{233}=2 \wp_{23}{ }^{3}+2 \wp_{33} \wp_{23} \wp_{22}+2 \mu_{9}+4 \wp_{23} \wp_{13}+2 \mu_{6} \wp_{23}+2 \mu_{3} \wp_{13} \\
& +2 \mu_{3} \wp_{23}^{2}+\mu_{3} \wp_{22} \wp_{33}, \quad[-9] \\
& \wp_{222} \wp_{333}=-\mu_{3} \wp_{23}{ }^{2}-4 \mu_{3} \wp_{13}+4 \mu_{3} \wp_{22} \wp_{33}-2 \wp_{23}{ }^{3}-8 \wp_{13} \wp_{23} \\
& +6 \wp_{33} \wp_{23} \wp_{22}-4 \wp_{33} \wp_{12}, \quad[-9] \\
& \wp_{223}{ }^{2}=4 \wp_{23}{ }^{2} \wp_{22}+4 \wp_{11}+4 \wp_{22} \wp_{13}+4 \mu_{6} \wp_{22}-4 \wp_{23} \wp_{12}-4 \mu_{3} \wp_{12} \\
& +\mu_{3}{ }^{2} \wp_{33}{ }^{2}-4 \mu_{6} \wp_{33}{ }^{2}+\frac{4}{3} \wp_{33} \wp_{1333}-8 \wp_{13} \wp_{33}{ }^{2}+4 \mu_{3} \wp_{23} \wp_{22},[-10] \\
& \wp_{133} \wp_{333}=-2 \wp_{22} \wp_{13}+\wp_{12} \wp_{23}+\frac{2}{3} \wp_{33} \wp_{1333}, \quad[-10] \\
& \wp_{233} \wp_{222}=2 \mu_{3} \wp_{12}-\frac{8}{3} \wp_{33} \wp_{1333}+2 \wp_{33} \wp_{22}^{2}+8 \mu_{6} \wp_{33}^{2}+16 \wp_{33}{ }^{2} \wp_{13} \\
& -2 \mu_{3}^{2} \wp_{33}^{2}+4 \wp_{23} \wp_{12}+\mu_{3} \wp_{23} \wp_{22}+2 \wp_{23}^{2} \wp_{22}, \quad[-10] \\
& \wp_{123} \wp_{333}=4 \wp_{33} \wp_{23} \wp_{13}+2 \mu_{3} \wp_{33} \wp_{13}-2 \wp_{22} \wp_{12}-\frac{1}{3} \wp_{23} \wp_{1333}+2 \wp_{33}^{2} \wp_{12}, \quad[-11] \\
& \wp_{223} \wp_{222}=8 \wp_{33} \wp_{13} \wp_{23}-\frac{2}{3} \mu_{3} \wp_{1333}+4 \mu_{9} \wp_{33}+4 \mu_{6} \wp_{33} \wp_{23}+4 \mu_{3} \wp_{33} \wp_{13} \\
& +2 \mu_{3} \wp_{22}^{2}-\frac{4}{3} \wp_{23} \wp_{1333}-\mu_{3}^{2} \wp_{33} \wp_{23}+4 \wp_{23} \wp_{22}{ }^{2}, \quad[-11] \\
& \wp_{233} \wp_{133}=2 \mu_{9} \wp_{33}+2 \mu_{3} \wp_{33} \wp_{13}+\frac{2}{3} \wp_{23} \wp_{1333}+\wp_{22} \wp_{12}, \quad[-11] \\
& \wp_{123} \wp_{233}=2 \wp_{33} \wp_{23} \wp_{12}+2 \mu_{3} \wp_{33} \wp_{12}-2 \wp_{33} \wp_{11}-\frac{1}{3} \wp_{22} \wp_{1333}+2 \wp_{33} \wp_{22} \wp_{13} \\
& +2 \mu_{3} \wp_{23} \wp_{13}+2 \wp_{13}^{2}+2 \mu_{6} \wp_{13}+2 \wp_{23}^{2} \wp_{13}+2 \mu_{12}, \quad[-12] \\
& \wp_{333} \wp_{122}=-2 \wp_{33} \wp_{22} \wp_{13}-\mu_{3} \wp_{23} \wp_{13}-2 \wp_{13} \wp_{23}{ }^{2}-6 \wp_{13}{ }^{2}-2 \mu_{6} \wp_{13}+\frac{2}{3} \wp_{22} \wp_{1333} \\
& +4 \wp_{33} \wp_{23} \wp_{12}+2 \mu_{3} \wp_{33} \wp_{12}-2 \wp_{33} \wp_{11}+2 \mu_{12}+\mu_{9} \wp_{23}, \quad[-12] \\
& \wp_{223} \wp_{133}=2 \mu_{3} \wp_{23} \wp_{13}+2 \wp_{23}^{2} \wp_{13}+2 \wp_{13}^{2}+2 \mu_{6} \wp_{13}-\mu_{3} \wp_{33} \wp_{12} \\
& -2 \wp_{33} \wp_{22} \wp_{13}+\frac{2}{3} \wp_{22} \wp_{1333}+2 \wp_{33} \wp_{11}+2 \mu_{12}, \quad[-12] \\
& \wp_{222}^{2}=-4 \mu_{3} \wp_{33} \wp_{12}+8 \wp_{33} \wp_{11}-4 \wp_{22} \wp_{1333}+24 \wp_{33} \wp_{22} \wp_{13} \\
& +4 \mu_{3} \wp_{23} \wp_{13}-8 \wp_{13}^{2}-4 \mu_{9} \wp_{23}-8 \wp_{13} \mu_{6}+4 \wp_{22}{ }^{3}+4 \mu_{3}^{2} \wp_{13} \\
& +\mu_{3}{ }^{2} \wp_{23}{ }^{2}-4 \mu_{6} \wp_{23}{ }^{2}-4 \mu_{3}{ }^{2} \wp_{33} \wp_{22}+16 \mu_{6} \wp_{33} \wp_{22}-8 \mu_{12}, \quad[-12] \\
& \wp_{223} \wp_{123}=-2 \wp_{23} \wp_{11}+2 \mu_{9} \wp_{22}+2 \wp_{13} \wp_{23} \wp_{22}+2 \mu_{3} \wp_{23} \wp_{12}+2 \wp_{12} \wp_{23}{ }^{2} \\
& +\frac{1}{3} \mu_{3} \wp_{33} \wp_{1333}-2 \mu_{3} \wp_{13} \wp_{33}^{2}-2 \mu_{9} \wp_{33}^{2}+2 \mu_{3} \wp_{22} \wp_{13}, \quad[-13] \\
& \wp_{133} \wp_{222}=4 \wp_{23} \wp_{22} \wp_{13}-\mu_{3} \wp_{23} \wp_{12}-2 \wp_{23}^{2} \wp_{12}-\frac{2}{3} \mu_{3} \wp_{33} \wp_{1333}+4 \mu_{3} \wp_{13} \wp_{33}{ }^{2} \\
& +4 \mu_{9} \wp_{33}^{2}+2 \mu_{3} \wp_{22} \wp_{13}+2 \wp_{33} \wp_{22} \wp_{12}, \quad[-13] \\
& \wp_{122} \wp_{233}=-\mu_{9} \wp_{22}+4 \wp_{13} \wp_{12}+2 \mu_{6} \wp_{12}-\frac{2}{3} \mu_{3} \wp_{33} \wp_{1333}+2 \wp_{33} \wp_{22} \wp_{12} \\
& +4 \mu_{3} \wp_{33}^{2} \wp_{13}+4 \mu_{9} \wp_{33}^{2}+2 \mu_{3} \wp_{23} \wp_{12}+2 \wp_{23}^{2} \wp_{12}-\mu_{3} \wp_{22} \wp_{13},
\end{aligned}
$$


J. C. Eilbeck et al.

$$
\begin{aligned}
& \wp_{333} \wp_{113}=-2 \wp_{12}^{2}-\frac{2}{3} \wp_{13} \wp_{1333}+6 \wp_{33} \wp_{13}^{2}+2 \mu_{6} \wp_{33} \wp_{13}+2 \wp_{33}^{2} \wp_{11} \\
& -2 \mu_{12} \wp_{33}-\mu_{9} \wp_{33} \wp_{23}, \quad[-14] \\
& \wp_{133}^{2}=\frac{4}{3} \wp_{13} \wp_{1333}+\wp_{12}^{2}-4 \wp_{33} \wp_{13}^{2}+4 \mu_{12} \wp_{33}, \quad[-14] \\
& \wp_{223} \wp_{122}=-2 \wp_{11} \wp_{22}+4 \wp_{23} \wp_{22} \wp_{12}+\frac{4}{3} \wp_{13} \wp_{1333}+2 \wp_{12}^{2}-8 \wp_{33} \wp_{13}^{2} \\
& +\mu_{3}\left(2 \wp_{22} \wp_{12}+4 \wp_{33} \wp_{23} \wp_{13}\right) \\
& -\frac{2}{3} \mu_{3} \wp_{23} \wp_{1333}+\mu_{3}^{2} \wp_{33} \wp_{13}-\frac{2}{3} \mu_{6} \wp_{1333}+4 \mu_{9} \wp_{33} \wp_{23} \\
& +\left(8 \mu_{12}+\mu_{3} \mu_{9}\right) \wp_{33}, \quad[-14] \\
& \wp_{123} \wp_{222}=2 \wp_{22}^{2} \wp_{13}+2 \wp_{23} \wp_{22} \wp_{12}-\frac{8}{3} \wp_{13} \wp_{1333}-2 \wp_{12}^{2}+16 \wp_{33} \wp_{13}{ }^{2} \\
& +\mu_{3}\left(2 \wp_{22} \wp_{12}-2 \wp_{33} \wp_{23} \wp_{13}+\frac{1}{3} \wp_{23} \wp_{1333}\right)+\left(8 \mu_{6}-2 \mu_{3}^{2}\right) \wp_{33} \wp_{13} \\
& -2 \mu_{9} \wp_{33} \wp_{23}, \quad[-14] \\
& \wp_{333} \wp_{112}=-2 \wp_{23} \wp_{13}^{2}+2 \mu_{6} \wp_{23} \wp_{13}+2 \wp_{33} \wp_{23} \wp_{11}-2 \mu_{12} \wp_{23}-\mu_{9} \wp_{23}{ }^{2} \\
& +\frac{4}{3} \wp_{12} \wp_{1333}-4 \mu_{3} \wp_{13}^{2}-4 \wp_{33} \wp_{13} \wp_{12}, \quad[-15] \\
& \wp_{113} \wp_{233}=2 \wp_{23} \wp_{13}^{2}+2 \wp_{33} \wp_{23} \wp_{11}-2 \mu_{12} \wp_{23}-\frac{2}{3} \wp_{12} \wp_{1333}+2 \mu_{3} \wp_{13}{ }^{2} \\
& +4 \wp_{33} \wp_{13} \wp_{12}-\mu_{9} \wp_{33} \wp_{22}+2 \mu_{6} \wp_{33} \wp_{12}+2 \mu_{9} \wp_{13}, \quad[-15] \\
& \wp_{123} \wp_{133}=2 \wp_{23} \wp_{13}{ }^{2}-2 \mu_{12} \wp_{23}+\frac{1}{3} \wp_{12} \wp_{1333}+2 \mu_{3} \wp_{13}{ }^{2}+2 \mu_{9} \wp_{13}, \quad[-15] \\
& \wp_{122} \wp_{222}=\frac{4}{3} \wp_{12} \wp_{1333}+8 \wp_{33} \wp_{13} \wp_{12}+4 \wp_{22}{ }^{2} \wp_{12}-2 \mu_{3} \wp_{13}{ }^{2}+2 \mu_{3} \wp_{33} \wp_{11}-\frac{2}{3} \mu_{3} \wp_{22} \wp_{1333} \\
& +4 \mu_{3} \wp_{33} \wp_{13} \wp_{22}+\mu_{3}^{2} \wp_{23} \wp_{13}+\left(-2 \mu_{3}^{2}+4 \mu_{6}\right) \wp_{33} \wp_{12}+\left(2 \mu_{3} \mu_{6}-8 \mu_{9}\right) \wp_{13} \\
& +6 \mu_{9} \wp_{33} \wp_{22}-2 \mu_{9} \wp_{23}{ }^{2}-\mu_{3} \mu_{9} \wp_{23}-2 \mu_{3} \mu_{12} . \quad[-15]
\end{aligned}
$$

The above equations describe the Jacobi variety as an algebraic variety, see also [10] where a general matrix construction is given. By eliminating odd powers with the aid of identities such as

$$
\wp_{333}^{2} \wp_{233}^{2}-\left(\wp_{333} \wp_{233}\right)^{2}=0
$$

one can obtain equations of the Kummer variety, $J /(u \rightarrow[-1] u)$.

\section{Acknowledgments}

This article was started during a visit by the authors to Tokyo Metropolitan University (TMU) in 2005, supported by Japan Society for the Promotion of Science (JSPS) grant 16540002. We would like to express our thanks to Prof. M. Guest of TMU who helped organize this visit. The work continued 
during a visit by VZE to Heriot-Watt University under the support of the Royal Society. Further work was done whilst three of the authors (JCE, VZE, and EP) were attending the programme in Nonlinear Waves at the Mittag-Leffler Institute in Stockholm in 2005, and we would like to thank Prof. H. Holden of Trondheim and the Royal Swedish Academy of Sciences for making this possible [EP, being then on leave from Boston University, is grateful for the National Security Agency (NSA) grant MDA904-031-0119 which supported her doctoral students who were performing related research]. The authors are also grateful for a number of useful discussions with Prof. A. Nakayashiki, and Drs. John Gibbons and Sadie Baldwin. In particular we are grateful to John Gibbons for pointing out the possibility of the relations described in Remarks 9.3 and 10.3. We are grateful to Mr. Matthew England for pointing out a number of typos in various versions of this manuscript. Some of the calculations described in this paper were carried out using Distributed Maple [29], and we are grateful to the author of this package, Prof. Wolfgang Schreiner of Research Institute for Symbolic Computation-Linz, for help and advice.

Finally, we would like to express special thanks to the referees for constructive suggestions to improve the article, in particular for pointing out some crucial gaps in the main theorems and for giving hints how to fill them.

\section{References}

[1] Baker, H. F. Abelian Functions. Cambridge, UK: Cambridge University Press, 1897.

[2] Baker, H. F. "On the hyperelliptic sigma functions." American Journal of Mathematics 20 (1898): 301-84.

[3] Baker, H. F. "On a system of differential equations leading to periodic functions." Acta Mathematica 27, no. 1 (1903): 135-56.

[4] Baker, H. F. Multiply Periodic Functions. Cambridge, UK: Cambridge University Press, 1907.

[5] Baldwin, S., and J. Gibbons. "Genus 4 trigonal reduction of the Benney equations." Journal of Physics A-Mathematical and General 39 (2006): 3607-39.

[6] Baldwin S., J. C. Eilbeck, J. Gibbons, and Y. Ônishi. “Abelian functions for purely trigonal curves of genus four." (2006): preprint arxiv.org/abs/math.AG/0612654.

[7] Buchstaber, V. M., and D. V. Leykin. "Polynomial Lie algebras." Functional Analysis and its Applications 36, no. 4 (2002): 267-80.

[8] Buchstaber, V. M., and D. V. Leykin. "Addition laws on Jacobian varieties of plane algebraic curves." Proceedings of the Steklov Institute of Mathematics 251 (2005): 54-126.

[9] Buchstaber, V. M., V. Z. Enolskii, and D. V. Leykin. "Kleinian functions, hyperelliptic Jacobians and applications." Reviews in Mathematics and Mathematical Physics 10 (1997): 1-125.

[10] Buchstaber, V. M., V. Z. Enolskii, and D. V. Leykin. “Uniformization of Jacobi varieties of trigonal curves and nonlinear equations." Functional Analysis and its Applications 34 (2000): 159-71.

[11] Burchnall, J. L., and T. W. Chaundy. "Commutative ordinary differential operators." Proceedings of the London Mathematical Society 118 (1923): 420-40. 
[12] Cassels, J. W. S., and E. V. Flynn. Prolegomena to a Middlebrow Arithmetic of Curves of Genus 2. London Mathematical Society Lecture Notes 230. Cambridge, UK: Cambridge University Press, 1996.

[13] Cho, K., and A. Nakayashiki. "Differential structure of Abelian functions." International Journal of Mathematics (2006): preprint arxiv.org/abs/math.AG/0604267.

[14] Eilbeck, J. C., and V. Z. Enolskii. "Bilinear operators and the power series for the Weierstrass $\sigma$ function." Journal of Physics A-Mathematical and General 33 (2000): 791-4.

[15] Eilbeck, J. C. "Weierstrass functions for higher genus curves." maintained by J. C. Eilbeck http://www.ma.hw.ac.uk/Weierstrass/ (accessed September 28, 2007).

[16] Eilbeck, J. C., V. Z. Enolskii, and D. V. Leykin. "On the Kleinian Construction of Abelian Functions of Canonical Algebraic Curves." In SIDE III-Symmetries and Integrable of Differences Equations, edited by D. Levi and O. Ragnisco, 121-38. Lecture Notes 25. Providence, RI: American Mathematical Society, 2000.

[17] Eilbeck, J. C., V. Z. Enolskii, and E. Previato. “On a generalized Frobenius-Stickelberger addition formula." Letters in Mathematical Physics 65 (2003): 5-17.

[18] Fay, J. D. Theta Functions on Riemann Surfaces. Lecture Notes in Mathematics 352. Berlin: Springer, 1973.

[19] Grant, D. “Formal groups in genus two." Journal Fur Die Reine Und Angewandte Mathematik 411 (1990): 96-121.

[20] Lang, S. Introduction to Algebraic Functions and Abelian Functions, Number 89 in Graduate Text in Mathematics, 2nd edn. Berlin: Springer, 1982.

[21] Matsutani, S., and E. Previato. "Jacobi inversion on strata of the Jacobian of the $C_{r s}$ curve $y^{r}=f(x) . "$ Moduli Spaces no. 41 (2006/2007): preprint http://www.mittagleffler.se/preprints/0607/.

[22] Mumford, D. Tata Lectures on Theta I. Progress in Mathematics 28. Boston, MA: Birkhäuser, 1983.

[23] Mumford, D. Abelian Varieties. Oxford: Oxford University Press, 1985.

[24] Ônishi, Y. "Complex multiplication formulae for hyperelliptic curves of genus three." Tokyo Journal Mathematics 21 (1998): 381-431.

[25] Ônishi, Y. "Abelian functions for trigonal curves of degree four and determinantal formulae in purely trigonal case." (2005): preprint arxiv.org/abs/math.NT/0503696.

[26] Ônishi, Y. "Determinant expressions for hyperelliptic curves." Proceedings of the Edinburgh Mathematical Society 48 (2005): 705-42.

[27] Shiga, H. "On the representation of the Picard modular function by $\theta$ constants I-II." Publications of the Research Institute for Mathematical Sciences Kyoto University 24 (1988): 311-60.

[28] Schilling, R. J. "Generalizations of the Neumann system: a curve theoretical approach II." Communications on Pure and Applied Mathematics 42 (1989): 409-42.

[29] Schreiner, W., C. Mittermaier, and K. Bosa. "Distributed Maple: Parallel computer algebra in networked environments." Journal of Symbolic Computation 35 (2003): 305-47. 